\title{
Local Knowledge and Use of Medicinal Plants in a Rural Community in the Agreste of Paraíba, Northeast Brazil
}

\author{
Ezequiel da Costa Ferreira ${ }^{1},{ }^{1}$ Maria da Glória Vieira Anselmo (iD, ${ }^{2}$ \\ Natan Medeiros Guerra $\left(\mathbb{D},{ }^{3}\right.$ Camilla Marques de Lucena ${ }^{(D)}{ }^{1}$ \\ Cattleya do Monte Pessoa Felix $\left(\mathbb{D},{ }^{1}\right.$ Rainer W. Bussmann $\left(\mathbb{D},{ }^{4}\right.$ \\ Narel Y Paniagua-Zambrana $\left(\mathbb{D},{ }^{4}\right.$ and Reinaldo Farias Paiva de Lucena ${ }^{5}{ }^{5}$ \\ ${ }^{1}$ Postgraduate Program in Development and Environment (PRODEMA), Ethnobiology and Environmental Sciences Laboratory, \\ Federal University of Paraíba, João Pessoa, Paraíba State, Brazil \\ ${ }^{2}$ Federal University of Paraíba, Master's Degree in Agronomy, Areia, Paraíba State, Brazil \\ ${ }^{3}$ Rural Federal University of the Semiarid, Doctoral Student, Plant Science, Mossoró, Rio Grande do Norte, Brazil \\ ${ }^{4}$ Ilia State University, Tbilisi, Georgia \\ ${ }^{5}$ Federal University of Mato Grosso do Sul, Institute of Biosciences, Ethnobotany Laboratory, \\ Research Group on Multidisciplinary and Socioecological Studies, Campo Grande, Mato Grosso do Sul, Brazil \\ Correspondence should be addressed to Reinaldo Farias Paiva de Lucena; reinaldo.lucena@ufms.br
}

Received 31 March 2021; Accepted 29 November 2021; Published 29 December 2021

Academic Editor: Maan B. Rokaya

Copyright (c) 2021 Ezequiel da Costa Ferreira et al. This is an open access article distributed under the Creative Commons Attribution License, which permits unrestricted use, distribution, and reproduction in any medium, provided the original work is properly cited.

\begin{abstract}
The use of medicinal plants is an important source of therapeutic resources in rural communities and the wide versatility of some species may attract interest for prospecting studies. The aim of this study was to record and analyze local knowledge and the use of medicinal plants in the rural community of Malícia, municipality of Araçagi, Paraíba State, Northeastern Brazil, applying quantitative methods to calculate the Relative Importance (RI) and the Informant Consensus Factor (ICF). Semistructured interviews were conducted with 46 heads of households. The interviews addressed questions about the used parts of the plants, therapeutic indications, and form of use. Therapeutic indications were classified into categories of body systems. The Relative Importance Index (RI) was calculated to verify the species versatility, and the Informant Consensus Factor (ICF) was calculated to verify the consensus of use among informants regarding the body systems. A total of 111 plant species were recorded, inside 101 genera and 47 families. Fabaceae (16 spp.), Lamiaceae, and Myrtaceae (each one with 7 spp.) were the most representative families. Mentha arvensis, Aloe vera, and Myracrodruon urundeuva had the highest RI. A high consensus of use was observed among the informants for neoplasms, nervous system diseases, and infectious and parasitic diseases. Leaves were the part most cited for medicinal use. Regarding the method of preparation, the decoction and the oral administration route stood out. Neoplasms and respiratory system diseases had the highest ICF values. The results indicate a diversified knowledge of the local pharmacopeia and the need for in-depth studies to corroborate the effectiveness of medicinal plants and to understand the dynamics of local knowledge.
\end{abstract}

\section{Introduction}

The use of plants for the treatment of diseases is ancient among human populations, considering the primary need of people to maintain basic care for their own health [1].

The concept of traditional medicine can be understood as a set of health practices, knowledge and beliefs, incorporating plants, animals, and minerals, as well as the use of spiritual, manual, and exercise therapies, which can be applied in isolation or in combination, to maintain wellbeing, treatment, prevention, or diagnosis of diseases [2].

Some authors, when classifying diseases mentioned by people, place categories of diseases not recognized by allopathic medicine, but forming part of the nosological 
universe of informants, as "cultural diseases" or "beliefs" $[3,4]$.

Even with the advent of allopathic medicine, the use of medicinal plants is still a valuable resource, especially in developing countries, where access to synthetic medicines is often not feasible due to high prices $[5,6]$, or by the lack of access to the biomedical health system, which often does not cover rural areas, requiring the use of medicinal plants for primary health care because they are more accessible [7].

Other issues involving the use of medicinal plants are the belief in this type of treatment and the search for drugs that do not present as many side effects as the allopathic ones $[6,8,9]^{\cdot}$ Even in cases where the biomedical health system is available, combinations between this and the traditional system for health treatments are common [7].

Ethnobotany and ethnopharmacology have often been used in research for the search for new drugs, often due to the low financial and time investment that these surveys demand. While ethnobotany is concerned with researching and documenting the relationships between people and plants, based on the human perception of plant resources, ethnopharmacology deals with the evaluation of traditional preparations used to treat diseases, seeking to test their efficacy [9-11]. Traditional knowledge about medicinal plants, in contrast to modern medicinal trends, tends to be orally transmitted and easily disappears with ethnobotanical and ethnopharmacological studies thus being a useful and necessary way of keeping this knowledge integrated with modern medicine [12]. A possible explanation for the progressive loss of traditional knowledge could be the fact that the advancement of allopathic medicine, as part of the globalization process, in some places may cause the local populations to become disinterested in traditional medicine, either by the younger generations losing interest in learning or the older ones in transmitting it [7-9, 12, 13].

There is also a trend in studies aiming to subsidize future research in search of new drugs, which may be more effective in the treatment of diseases, and with fewer side effects [9].

Given the context of the need to preserve traditional knowledge about the use of medicinal plants, quite a few ethnobotanical studies have been carried out in Brazil, including some in the state of Paraíba [14-21]. These studies showed, however, a greater emphasis on diseases related to the respiratory and digestive systems and encountered anything from 36 to 140 species, mostly indicating greater diversity in the families Fabaceae and Euphorbiaceae. This lies in agreement with a higher register of these families in floristic and phytosociological surveys carried out in the region [22-24]. These studies are spread over different types of traditional communities in Brazil, e.g., farmer communities [25-27], indigenous populations [28,29], quilombolas [30,31], riparian populations [32], and caiçaras [33].

Works of this genre have also been developed in different areas and communities around the world, as a few diverse examples serve healers in Guinea-Bissau [34], valley communities in the Italian Alps [35], indigenous villages in the Limu Mountains, China [36], or indigenous communities in Mexico [37], and children in a school in Argentina [38].
Different hypotheses have been proposed and tested in ethnobotanical studies with medicinal plants such as utilitarian redundancy, which seeks to explain the resilience of local medical systems $[39,40]$; the hypothesis of diversification, which proposes explaining the incorporation of exotic species to local pharmacopeias through a possible increase in bioactive compounds that these plants can bring [41]; or the hypothesis of appearance, which seeks to explain how medicinal plants are selected considering their chemical composition or ecological characteristics, classifying them as apparent and not apparent, based on the thought that, from a chemical perspective, herbaceous (or nonwoody) species are considered to be apparent, assuming that they contain a higher concentration of metabolites, whereas shrubs and trees (or woody) are considered as nonapparent [27,42]. Equally, from an ecological perspective, species easily visible due to their size (such as trees and shrubs) or life-cycle characteristics are more apparent, while small herbaceous species are considered not apparent in the initial stages of succession [42-45]. In both cases, it is inferred that species considered to be apparent are those that are more frequently collected and used more recurrently.

Considering the high level of use of medicinal plants in developing countries and the growing interest in the field of herbal medicine, it is also necessary to take a closer look at the conservation of these resources, because many communities are dependent on their availability, and many species of medicinal plants have by now appeared on lists of endangered species, creating a need to find practices for a more sustainable use [46].

From all of these varied perspectives, studies on medicinal plants are of great importance in Brazil, given their great biodiversity, territorial dimension, and progressive loss of traditional knowledge due to the impacts of globalization on traditional populations.

The present study aimed to record and analyze local knowledge and the use of medicinal plants in the rural Malícia community of Araçagi, Paraíba, Northeastern Brazil, seeking to verify the local importance of the species and how widespread is the local knowledge about these species for categories of medicinal use. We started with the following questions. What is the number of species known locally for medicinal use? What is the origin and way of life of the species used? Are there differences between the most cited species and the most versatile? Which medicinal categories have the greatest consensus for local use? What parts of plants are used and how are they prepared for medicinal use?

The following study assumes the following hypotheses: (a) in the study area there is a greater knowledge of exotic plants than of the native ones, both in the number of species and in the diversity of uses; (b) there is a correspondence between the most cited species and the most versatile; (c) there is a greater consensus of use for the medicinal categories with the highest number of citations. A point to be highlighted is that the studied community is located in an ecotone area, in the transition between the Mata Atlântica and the Caatinga, where we found species from both biomes, which makes the region with peculiar characteristics. 


\section{Materials and Methods}

2.1. Geoenvironmental Characterization. The municipality of Araçagi is located in the mesoregion of the Agreste and microregion of Guarabira, in the state of Paraíba, Northeastern Brazil (Figure 1). It is located between $06^{\circ} 51^{\prime} 10^{\prime \prime} \mathrm{S}$ and $35^{\circ} 22^{\prime} 51^{\prime \prime} \mathrm{E}$ [47], with an altitude of $57 \mathrm{~m}$ at the municipal headquarters [48]. The region is approximately $64 \mathrm{~km}$ from the state capital, João Pessoa, and borders the municipalities of Duas Estradas, Curral de Cima, and Sertãozinho to the north, Mulungú, Sapé, Mari, and Capim to the south, Cuité de Mamanguape, Mamanguape, and Itapororoca to the east, and Guarabira and Pirpirituba to the west. The region has a population of approximately 17224 inhabitants, with 6804 inhabitants in an urban area and 10420 inhabitants in a rural area, with 8574 men and 8650 women. Its territory is $231155 \mathrm{~km}^{2}$, with a population density of 74.51 inhabitants per $\mathrm{km}^{2}$ [47].

The municipality is part of the geoenvironmental unit of Serrotes, Inselbergs, and Maciços residuais. The vegetation is Caatinga, with small areas of Deciduous Forest. The climate regime is hot, with winter rains from February to August and average annual precipitation around $750 \mathrm{~mm}$ [48].

The community of Malícia is about $9 \mathrm{~km}$ from the municipal urban center and has a football field, a market, and some small points of commerce.

Access to primary education and basic health is only available in the neighboring community (Agrovila Mulunguzinho), which has a Primary School I (Municipal School of Primary Education João Dutra de Araújo) and a Basic Health Unit, where the residents have access to medical and dental care.

The economy is based on small trading and subsistence farming, with special emphasis on the cultivation of beans, maize, fava, yam, cassava, and the raising of cattle, goats, sheep, and pigs. Some community residents work on a nearby farm, others as day laborers on pineapple plantations around the community. Most of the native forest areas close to the community have been deforested for agricultural practice, more intensely in the last decade, due to the diffusion of pineapple monoculture in the region.

2.2. Collection of Ethnobotanical Data. Interviews were conducted with household heads (men and women) [49]. Three visits were made throughout the community between June and August 2015, with the aim of interviewing 100\% of the informants. Ultimately 46 people, of whom 17 were men and 29 were women, participated in the interviews.

Before the interview with each person, the research objectives were clearly explained, and each participant was invited to sign the free and informed consent form, requested by the National Health Council, through the Research Ethics Committee (Resolution No. 196/1996) of the University Hospital Lauro Wanderley (CEP/HULW) of the University Federal of Paraíba.

The data collection form involved questions regarding medicinal plants, their used parts, method of preparation, route of administration, disease treated, and socioeconomic information such as age, profession, marital status, and level of schooling of the participants. The names of the species cited were recorded according to the pronunciation of the informants.

Species available with fertile material in local vegetation were collected and herborized in the field and then identified and incorporated in the Jaime Coelho de Moraes Herbarium (EAN), of the Federal University of Paraíba (UFPB), at the Agricultural Sciences Center (CCA).

2.3. Data Analysis. Each species was classified according to its place of origin as exotic (from outside Brazil) or native (from Brazil). The plant life forms were classified as herbaceous, shrub, tree, and liana.

The therapeutic indications mentioned by the informants were classified in categories except for cases of insufficient information for such a classification and body systems according to the International Classification of Diseases, version 2015 [50]. Based on this classification, the NBS (number of body systems) and NP (number of properties) were calculated for each species, with the following formula of Bennett and Prance [51]:NBS = NBSS/NBSVS. NBS refers to the number of body systems, resulting from the division of the number of body systems treated by a particular species (NBSS) by the total number of body systems treated by the most versatile species (NBSVS), with species used for a greater diversity of body systems being considered more versatile.

For NP, the following formula was used: $\mathrm{NP}=\mathrm{NPS} / \mathrm{NPVS}$. Here, NP is the number of properties, resulting from the division of the number of properties attributed to a species (NPS), by the number of properties attributed to the most versatile species (NPVS), with species with the highest number of properties considered more versatile.

The Relative Importance (RI) was then calculated for each species based on the following formula [51]: $\mathrm{RI}=\mathrm{NBS}+\mathrm{NP}$.

This method emphasizes the species that present greater versatility, i.e., those that present a greater diversity of uses. It represents a quantitative method that does not suffer the direct influence of the number of citations for a certain species, but of the diversity of applications for that species. The maximum value that can be obtained by the calculation is 2; an RI near this value indicates a greater versatility of a species.

The therapeutic properties were grouped into 17 categories: external causes of morbidity and mortality, skin and subcutaneous tissue disorders, ear diseases, diseases of the blood and hematopoietic organs, cardiovascular system diseases, diseases of the digestive system, diseases of the genitourinary system, nervous system disorders, osteomuscular and connective tissue diseases, respiratory system diseases, eye diseases, endocrine, nutritional, and metabolic diseases, infectious and parasitic diseases, injury, poisoning and some other consequences of external causes, neoplasms, symptoms and signs not elsewhere classified, and mental and behavioral disorders [50]. 


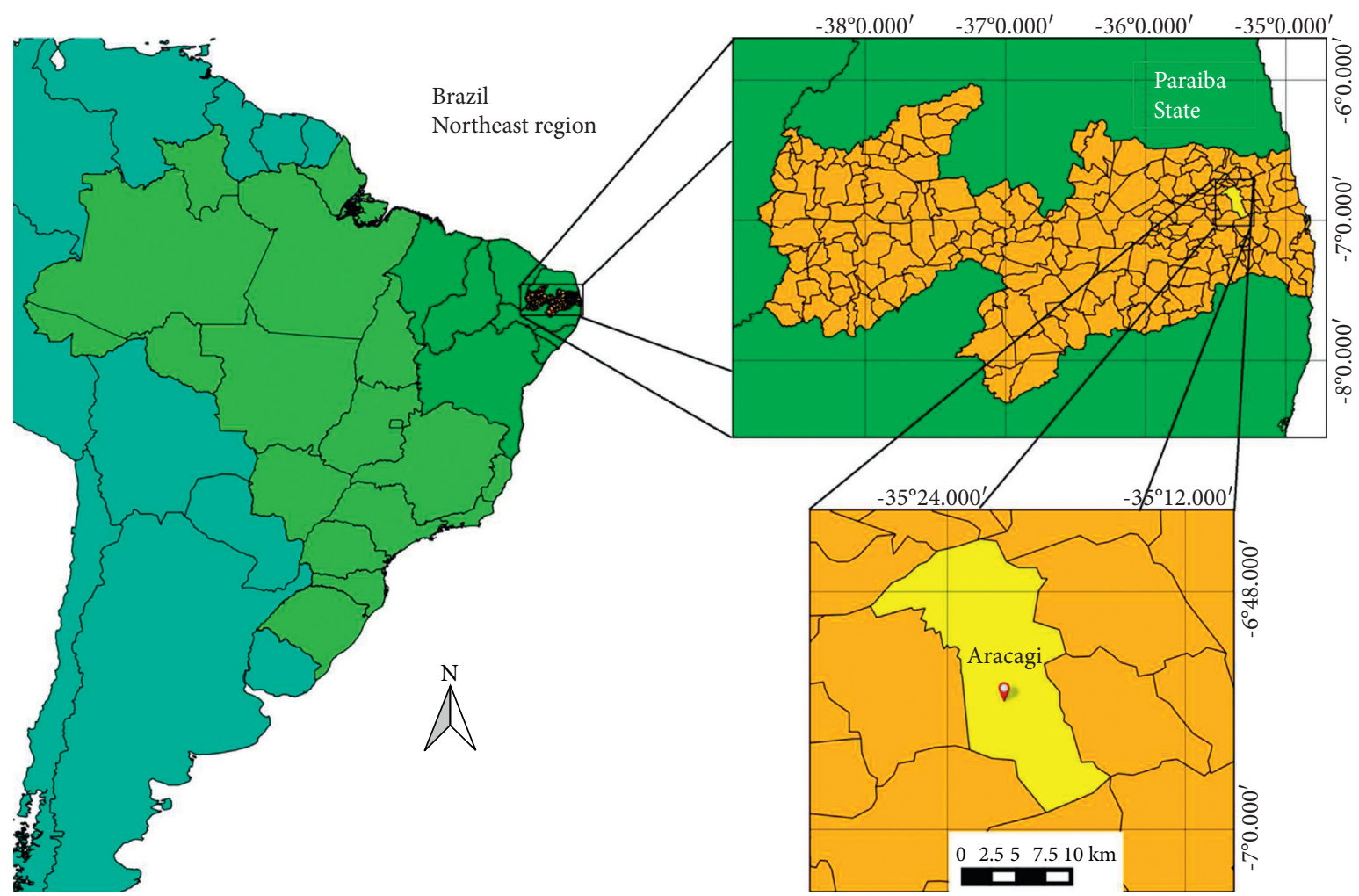

Figure 1: Location of the study area. Municipality of Araçagi, state of Paraíba, the northeastern region of Brazil.

In order to quantify the most important body systems, the Informants Consensus Factor (ICF) was calculated using the formula of Trotter and Logan [52]: $\mathrm{ICF}=$ nar $-\mathrm{na} / \mathrm{nar}-1$. Here, nar refers to the sum of the uses mentioned by each informant for a given category; na refers to the number of species cited in the category. The maximum value of the ICF is 1 , and the proximity of this value indicates that the informants present a consensus for the category observed.

\section{Results and Discussion}

3.1. Identified Plants. A total of 111 plant species were mentioned, of which 1 was identified only at the family level and 109 species were identified at least at the genus level, with 1 undetermined species (Table 1). The species identified are distributed in 101 genera and 47 families. The most significant were Fabaceae (16 spp.), Lamiaceae (7 sp.), and Myrtaceae (7 spp.).

In the state of Piauí, Brazil, only 57 species for medicinal use were observed [53]. This difference may be related to a lower number of informants (31, compared to 46 here interviewed). On the other hand, studies in other parts of the world have recorded a greater number of species, such as Guinea-Bissau (218 sp.) [34], as a consequence of a review of data obtained from healers in different indigenous communities during 17 years, in a riparian community in Brazil (309 sp.) [54] and in a quilombola community in Brazil (133 sp.) [55], which may be related to cultural factors, due to the strong belief in the healing power of plants affirmed by people.
When comparing the results of the present study with those performed in other biomes, we found that, e.g., in the Peruvian Amazon, there was a high representation for Fabaceae with 23 sp., followed by Araceae and Rubiaceae (both with 20 sp.) [56], whereas Lamiaceae and Myrtaceae (both with $4 \mathrm{sp}$.) were rare. In another Cerrado area, the most important families were Lamiaceae (10 sp.) and Asteraceae (7) [57]. In an area of Atlantic Forest, the highlights were Asteraceae (18 sp.), Lamiaceae (10 sp.), and Myrtaceae (9) [58].

The families Fabaceae, Lamiaceae, and Myrtaceae were also very important in a large study in the state of Piauí, Brazil [53], where Fabaceae presented $8 \mathrm{sp}$. and Lamiaceae and Myrtaceae showed both $5 \mathrm{sp}$. In Cariri Paraibano, the predominance of Fabaceae (16 sp.), Asteraceae (11 sp.), and Solanaceae (9 sp.) [59] was observed, and in the semiarid Bahia, Fabaceae (11 sp.), Asteraceae (6 sp.), and Anacardiaceae (5 sp.) [60] were the most important families.

The high number of Asteraceae and Lamiaceae in these studies might be explained by their adaptation to both tropical and temperate environments and by their cosmopolitan distribution [61]. On the other hand, the higher number of Fabaceae in the present study could be explained as maintenance of the knowledge about native species, since only one species of this family was exotic.

3.2. Life Form, Origin, and Endemism. There were 43 herbaceous plants, 36 trees, 19 shrubs, and 11 lianas. Of all plants, 56 species were natives of which 44 species were nonendemic natives, 10 endemic natives, and 2 natives 
TABLE 1: Data on medicinal plants: life forms, origin and endemism, parts used, and form of use, medicinal uses, number of citations, and Relative Importance.

\begin{tabular}{|c|c|c|c|c|c|c|c|c|}
\hline $\begin{array}{l}\text { Family/Scientific name/ } \\
\text { Voucher }\end{array}$ & $\begin{array}{l}\text { Local name/ } \\
\text { English name }\end{array}$ & $\begin{array}{l}\text { Life } \\
\text { form }\end{array}$ & $\begin{array}{l}\text { Origin and } \\
\text { endemism }\end{array}$ & $\begin{array}{l}\text { Parts } \\
\text { used }\end{array}$ & $\begin{array}{c}\text { Form of } \\
\text { use }\end{array}$ & Uses & NC & RI \\
\hline \multicolumn{9}{|l|}{ Adoxaceae } \\
\hline $\begin{array}{l}\text { Sambucus australis Cham. } \\
\text { \& Schltdl. } \\
\text { Amaranthaceae }\end{array}$ & Sabugueira & $\mathrm{S}$ & NEN & $\mathrm{Fl} / \mathrm{Lf}$ & $\begin{array}{l}\mathrm{Dc} / \mathrm{If} / \\
\mathrm{Lb}\end{array}$ & $\begin{array}{l}\text { Headache/Body aches/Fever/Flu/ } \\
\text { Cough }\end{array}$ & 17 & 0.52 \\
\hline $\begin{array}{l}\text { Alternanthera brasiliana } \\
\text { (L.) Kuntze }\end{array}$ & Anador & $\mathrm{H}$ & NEN & $\mathrm{Fl}$ & If/Dc & Headache/Fever & 2 & 0.24 \\
\hline Beta vulgaris $\mathrm{L}$. & Beterraba/Beet & $\mathrm{H}$ & EC & Ro & $\mathrm{Lb}$ & Flu & 1 & 0.19 \\
\hline $\begin{array}{l}\text { Dysphania ambrosioides } \\
\text { (L.) Mosyakin \& Clemants } \\
\text { (25.583) }\end{array}$ & Mentruz & $\mathrm{H}$ & ENT & $\mathrm{Lf} / \mathrm{Ei}$ & $\begin{array}{l}\mathrm{Dc} / \mathrm{Nt} / \\
\mathrm{If} / \mathrm{Lb} / \mathrm{Jc}\end{array}$ & $\begin{array}{c}\text { Blood thinning/Indigestion/ } \\
\text { Bronchitis/Tummy ache/Headache/ } \\
\text { Stomach/Expectoration/Fever/Flu/ } \\
\text { Cough/Ulcer/Verminosis }\end{array}$ & 59 & 1.29 \\
\hline \multicolumn{9}{|l|}{ Amaryllidaceae } \\
\hline Allium cepa L. (25.558) & Cebola/Onion & $\mathrm{H}$ & $\mathrm{EC}$ & $\mathrm{Bb} / \mathrm{Ei}$ & $\begin{array}{l}\text { If/Sy/ } \\
\text { Mc }\end{array}$ & $\begin{array}{c}\text { Indigestion/Flu/Hoarseness/Cough/ } \\
\text { Verminosis/Stroke }\end{array}$ & 8 & 1 \\
\hline $\begin{array}{l}\text { Allium sativum L. } \\
\text { Anacardiaceae }\end{array}$ & Alho/Garlic & $\mathrm{H}$ & $\mathrm{EC}$ & $\mathrm{Bl}$ & $\mathrm{Nt} / \mathrm{Dc}$ & Snake bite/Flu & 2 & 0.38 \\
\hline $\begin{array}{l}\text { Anacardium occidentale } \\
\text { L. (25.577) }\end{array}$ & $\begin{array}{l}\text { Cajueiro/cashew } \\
\text { tree }\end{array}$ & $\mathrm{T}$ & NEN & $\begin{array}{l}\mathrm{Ba} / \mathrm{Ib} / \\
\mathrm{Fr} / \mathrm{Sd}\end{array}$ & $\begin{array}{c}\mathrm{Dc} / \mathrm{Mc} / \\
\mathrm{Jc}\end{array}$ & $\begin{array}{l}\text { Healing/Inflamed tooth/infection/ } \\
\text { Inflammation/Inside inflammation/ } \\
\text { Injury/Snake bite/Burn }\end{array}$ & 16 & 0.95 \\
\hline Mangifera indica $\mathrm{L}$. & Manga/Mango & $\mathrm{T}$ & EC & Lf & $\mathrm{Dc} / \mathrm{If}$ & $\begin{array}{l}\text { Diabetes/Cholesterol/Malaise } \\
\text { Healing/Eczema/Expectoration/ }\end{array}$ & 3 & 0.43 \\
\hline $\begin{array}{l}\text { Myracrodruon urundeuva } \\
\text { Allemão }\end{array}$ & Aroeira & $\mathrm{T}$ & NEN & $\begin{array}{l}\mathrm{Ba} / \mathrm{Ib} / \\
\mathrm{Lf}\end{array}$ & $\begin{array}{l}\mathrm{Dc} / \mathrm{Gf} / \\
\mathrm{If} / \mathrm{Lb} / \\
\mathrm{Mc}\end{array}$ & $\begin{array}{l}\text { Infection/Inflammation/Gum } \\
\text { inflammation/Uterine inflammation/ } \\
\text { Urinary inflammation/Injury/Bruise/ } \\
\text { Cough }\end{array}$ & 27 & 1.52 \\
\hline \multicolumn{9}{|l|}{ Annonaceae } \\
\hline $\begin{array}{l}\text { Annona muricata } \\
\text { L. }(25.593)\end{array}$ & Graviola & $\mathrm{T}$ & $\mathrm{EC}$ & Lf/Sd & Dc & Diabetes/Thrombosis & 2 & 0.38 \\
\hline $\begin{array}{l}\text { Xylopia frutescens Aubl. } \\
\text { Apiaceae }\end{array}$ & Imbira & $\mathrm{T}$ & NEN & $\mathrm{Sd}$ & Dc & Tummy ache (abdominal pain) & 1 & 0.19 \\
\hline Daucus carota $\mathrm{L}$. & $\begin{array}{l}\text { Cenoura/Carrot } \\
\text { Coentro }\end{array}$ & $\mathrm{H}$ & EC & Ro & $\mathrm{Jc}$ & Intestinal detoxification & 1 & 0.19 \\
\hline Eryngium foetidum L. & $\begin{array}{l}\text { Maranhão/ } \\
\text { Maranhão } \\
\text { cilantro }\end{array}$ & $\mathrm{H}$ & NEN & Lf & Dc & Stroke & 1 & 0.19 \\
\hline $\begin{array}{l}\text { Pimpinella anisum } \\
\text { L. (25.596) }\end{array}$ & Erva doce/Fennel & $\mathrm{H}$ & $\mathrm{EC}$ & $\mathrm{Sd}$ & $\begin{array}{l}\text { Dc/Gf/ } \\
\text { If }\end{array}$ & $\begin{array}{l}\text { Tranquilizer/Pain/Tummy ache } \\
\text { (abdominal pain)/Indigestion/ } \\
\text { Insomnia/Polycystic ovary }\end{array}$ & 8 & 0.86 \\
\hline $\begin{array}{l}\text { Apocynaceae } \\
\text { Catharanthus roseus }(L) \\
\text { G. Don }(25.579)\end{array}$ & $\begin{array}{l}\text { Boa noite/Rose } \\
\text { periwinkle } \\
0.67\end{array}$ & $\mathrm{H}$ & EC & $\mathrm{Fl} / \mathrm{Ro}$ & $\begin{array}{l}\mathrm{Dc} / \mathrm{Nt} / \\
\mathrm{If} / \mathrm{Lb}\end{array}$ & $\begin{array}{l}\text { Headache/Toothache/Fever/Flu/ } \\
\text { Cough }\end{array}$ & 11 & \\
\hline Plumeria rubra $\mathrm{L}$. & $\begin{array}{l}\text { Jasmim vapor/ } \\
\text { Jasmine vapor }\end{array}$ & $\mathrm{S}$ & $\mathrm{EC}$ & Lt & $\mathrm{Nt}$ & Injury & 1 & 0.19 \\
\hline $\begin{array}{l}\text { Arecaceae } \\
\text { Cocus nucifera L (25.597) } \\
\text { Asparagaceae }\end{array}$ & Côco roxo & $\mathrm{T}$ & NEN & Lf & Dc & Jaundice & 1 & 0.19 \\
\hline $\begin{array}{l}\text { Aloe vera (L.) Burm. f } \\
(25.553)\end{array}$ & Babosa & $\mathrm{H}$ & $\mathrm{EC}$ & Lf & $\begin{array}{l}\mathrm{Dc} / \mathrm{Gf} / \\
\mathrm{Nt} / \mathrm{Lb} / \\
\mathrm{Jc} / \mathrm{Sp}\end{array}$ & $\begin{array}{c}\text { Cancer/Lumb/Acne/Expectoration/ } \\
\text { Furuncle/Gastritis/Flu/Hemorrhoids/ } \\
\text { Inflammation/Skin mark/Prostate/ } \\
\text { Cough/Ulcer }\end{array}$ & 23 & 1.62 \\
\hline \multicolumn{9}{|l|}{ Asteraceae } \\
\hline $\begin{array}{l}\text { Acanthospermum } \\
\text { hispidum DC (25.568) }\end{array}$ & $\begin{array}{l}\text { Espinho de } \\
\text { cigano/Gypsy- } \\
\text { Thorn }\end{array}$ & $\mathrm{H}$ & NEN & Ro & $\begin{array}{l}\mathrm{Dc} / \mathrm{If} / \\
\mathrm{Lb}\end{array}$ & Inflamed tooth/Flu/Kidney/Cough & 4 & 0.76 \\
\hline $\begin{array}{l}\text { Matricaria chamomilla } \\
\text { (L.) Rauschert }\end{array}$ & $\begin{array}{l}\text { Camomila/ } \\
\text { Chamomile }\end{array}$ & $\mathrm{H}$ & EC & $\mathrm{Fl}$ & Dc/If & Nerves/Tranquilizer & 3 & 0.19 \\
\hline
\end{tabular}


TABle 1: Continued.

\begin{tabular}{|c|c|c|c|c|c|c|c|c|}
\hline $\begin{array}{l}\text { Family/Scientific name/ } \\
\text { Voucher }\end{array}$ & $\begin{array}{l}\text { Local name/ } \\
\text { English name }\end{array}$ & $\begin{array}{l}\text { Life } \\
\text { form }\end{array}$ & $\begin{array}{l}\text { Origin and } \\
\text { endemism }\end{array}$ & $\begin{array}{l}\text { Parts } \\
\text { used }\end{array}$ & $\begin{array}{c}\text { Form of } \\
\text { use }\end{array}$ & Uses & $\mathrm{NC}$ & RI \\
\hline Helianthus annuus L. & $\begin{array}{l}\text { Girassol/ } \\
\text { Sunflower }\end{array}$ & $\mathrm{H}$ & $\mathrm{EC}$ & Sd & $\mathrm{Dc} / \mathrm{If}$ & Stroke & 2 & 0.19 \\
\hline $\begin{array}{l}\text { Bixaceae } \\
\text { Bixa orellana } \mathrm{L} . \\
\text { Boraginaceae }\end{array}$ & Açafrão & S & NEN & $\mathrm{Sd}$ & Mc & Bruise & 2 & 0.19 \\
\hline $\begin{array}{l}\text { Heliotropium indicum } \\
\text { L. }(25.598) \\
\text { Brassicaceae }\end{array}$ & Fedegoso & $\mathrm{H}$ & NEN & Ro & $\mathrm{Lb}$ & Expectorant/Cough & 2 & 0.38 \\
\hline Brassica oleracea L. & $\begin{array}{c}\text { Couve/ } \\
\text { Cauliflower }\end{array}$ & $\mathrm{H}$ & $\mathrm{EC}$ & Lf & Jc & Intestinal detoxification & 1 & 0.19 \\
\hline $\begin{array}{l}\text { Bromeliaceae } \\
\text { Ananas comosus (L.) } \\
\text { Merril }(25.560)\end{array}$ & $\begin{array}{l}\text { Abacaxi/ } \\
\text { Pineapple }\end{array}$ & $\mathrm{H}$ & EN & Fr & $\mathrm{Lb}$ & Bronchitis/Expectoration/Flu/Cough & 8 & 0.48 \\
\hline $\begin{array}{l}\text { Tillandsia recurvata (L.) L } \\
(25.577) \\
\text { Cactaceae }\end{array}$ & Samambaia & $\mathrm{H}$ & NEN & $\mathrm{Ei}$ & Dc & Jaundice & 1 & 0.19 \\
\hline Cereus jamacaru DC. & Cardeiro & $\mathrm{T}$ & EN & St & Mc & Kidney stones & 1 & 0.19 \\
\hline $\begin{array}{l}\text { Nopalea cochenillifera (L.) } \\
\text { Salm-Dyck (25.588) } \\
\text { Caricaceae }\end{array}$ & $\begin{array}{l}\text { Palma/Forage } \\
\text { cactus }\end{array}$ & $\mathrm{S}$ & ENT & St & $\mathrm{Mc}$ & Kidney stones & 1 & 0.19 \\
\hline $\begin{array}{l}\text { Carica papaya } \mathrm{L} . \\
\text { Caryophyllaceae }\end{array}$ & Mamão/Papaya & S & ENT & Fr & $\mathrm{Lb}$ & Flu/Cough & 4 & 0.38 \\
\hline $\begin{array}{l}\text { Dianthus caryophyllus } \\
\text { L. (25.563) } \\
\text { Cleomaceae }\end{array}$ & $\begin{array}{l}\text { Cravo branco/ } \\
\text { Carnation }\end{array}$ & $\mathrm{H}$ & $\mathrm{EC}$ & $\mathrm{Fl}$ & $\mathrm{Dc}$ & Asthma & 1 & 0.19 \\
\hline $\begin{array}{l}\text { Tarenaya aculeata }(\mathrm{L} .) \\
\text { Soares Neto \& roalson }\end{array}$ & Mussambê & $\mathrm{H}$ & NEN & $\mathrm{Fl} / \mathrm{Ro}$ & $\mathrm{Dc} / \mathrm{Lb}$ & Expectoration/Flu/Cough & 9 & 0.43 \\
\hline $\begin{array}{l}\text { Convolvulaceae } \\
\text { Ipomoea asarifolia (Desr.) } \\
\text { Roem. \& Schult (25.575) }\end{array}$ & Salsa & $\mathrm{H}$ & NEN & $\mathrm{Lf} / \mathrm{Ei}$ & $\mathrm{Dc} / \mathrm{Nt}$ & Inflammation/Healing & 3 & 0.38 \\
\hline $\begin{array}{l}\text { Operculina hamiltoni (G.) } \\
\text { Don. F. Austin \& Stapies } \\
\text { Costaceae }\end{array}$ & Batata de purga & $\mathrm{L}$ & NEN & $\mathrm{Tb}$ & Mc & Lump & 1 & 0.19 \\
\hline Costus spicatus (jacq.) Sw. & Cana do brejo & $\mathrm{H}$ & $\mathrm{N} *$ & $\begin{array}{l}\mathrm{Lf} / \mathrm{Ei} / \\
\text { Ro }\end{array}$ & $\mathrm{Dc} / \mathrm{If}$ & $\begin{array}{l}\text { Prostate/Kidney/Urinary } \\
\text { Inflammation }\end{array}$ & 5 & 0.29 \\
\hline $\begin{array}{l}\text { Crassulaceae } \\
\text { Kalanchoe crenata } \\
\text { (Andrews) Haw (25.585) } \\
\text { Cucurbitaceae }\end{array}$ & Saião & $\mathrm{H}$ & ENT & Lf & $\begin{array}{l}\mathrm{Dc} / \mathrm{Nt} / \\
\mathrm{Lb} / \mathrm{Jc}\end{array}$ & Pain/Gastritis/Flu/Cough/Ulcer & 17 & 0.67 \\
\hline $\begin{array}{l}\text { Apodanthera congestiflora } \\
\text { Cogn. }\end{array}$ & Cabeça de nego & $\mathrm{L}$ & NEN & $\mathrm{Tb}$ & Dc & Itch & 1 & 0.19 \\
\hline $\begin{array}{l}\text { Citrullus lanatus (Thunb.) } \\
\text { Matsum. \& Nakai }\end{array}$ & $\begin{array}{c}\text { Melancia/ } \\
\text { Watermelon }\end{array}$ & $\mathrm{L}$ & $\mathrm{EC}$ & $\mathrm{Fr} / \mathrm{Sd}$ & $\mathrm{Dc} / \mathrm{Nt}$ & $\begin{array}{c}\text { Conjunctivitis/Malaise/Prostate/ } \\
\text { Urine stimulation }\end{array}$ & 5 & 0.76 \\
\hline Cucumis anguria $\mathrm{L}$. & Maxixe & $\mathrm{L}$ & NEN & Fr & $\mathrm{Nt}$ & Cough & 1 & 0.19 \\
\hline Fevillea trilobata $\mathrm{L}$. & Gindiroba & $\mathrm{L}$ & NEN & $\mathrm{Sd}$ & $\mathrm{Jc}$ & Tiredness/Sinusitis/Constipation & 3 & 0.43 \\
\hline Luffa operculata Cong. & Cabacinha & $\mathrm{L}$ & NEN & $\mathrm{Fr}$ & $\mathrm{Dc}$ & Constipation/Lump & 2 & 0.38 \\
\hline $\begin{array}{l}\text { Momordica charantia } \\
\text { L. }(25.590) \\
\text { Euphorbiaceae }\end{array}$ & $\begin{array}{l}\text { Melão de São } \\
\text { caetano }\end{array}$ & $\mathrm{L}$ & ENT & $\mathrm{Lf} / \mathrm{Fr}$ & $\mathrm{Nt} / \mathrm{Jc}$ & Itch/Hemorrhoids/Verminosis & 7 & 0.43 \\
\hline $\begin{array}{l}\text { Cnidoscolus urens (L.) } \\
\text { Arthur (25.561) }\end{array}$ & Urtiga branca & S & NEN & Ro & $\begin{array}{c}\text { Dc/Gf/ } \\
\text { Mc }\end{array}$ & $\begin{array}{l}\text { Expectoration/Infection/ } \\
\text { Inflammation/Prostate }\end{array}$ & 9 & 0.62 \\
\hline Croton jacobinensis Baill. & Marmeleiro & S & EN & $\mathrm{Ba} / \mathrm{Lt}$ & $\mathrm{Nt} / \mathrm{Mc}$ & $\begin{array}{l}\text { Healing/Tummy ache (abdominal } \\
\text { pain)/Staunch bleeding }\end{array}$ & 3 & 0.43 \\
\hline $\begin{array}{l}\text { Jatropha mollissima } \\
\text { (Pohl) Baill (25.595) }\end{array}$ & Pinhão bravo & $S$ & NEN & $\mathrm{Lt}$ & $\mathrm{Nt}$ & Snake bite & 1 & 0.19 \\
\hline Manihot esculenta Crantz & $\begin{array}{l}\text { Macaxeira/ } \\
\text { Manioc }\end{array}$ & $S$ & NEN & Lf & $\mathrm{Nt}$ & Conjunctivitis & 1 & 0.19 \\
\hline $\begin{array}{l}\text { Ricinus communis } \\
\text { L. }(25.549) \\
\text { Fabaceae }\end{array}$ & Carrapateira & $S$ & ENT & St & As & Healing/Eczema & 2 & 0.38 \\
\hline
\end{tabular}


TABle 1: Continued.

\begin{tabular}{|c|c|c|c|c|c|c|c|c|}
\hline $\begin{array}{l}\text { Family/Scientific name/ } \\
\text { Voucher }\end{array}$ & $\begin{array}{l}\text { Local name/ } \\
\text { English name }\end{array}$ & $\begin{array}{l}\text { Life } \\
\text { form }\end{array}$ & $\begin{array}{l}\text { Origin and } \\
\text { endemism }\end{array}$ & $\begin{array}{l}\text { Parts } \\
\text { used }\end{array}$ & $\begin{array}{l}\text { Form of } \\
\text { use }\end{array}$ & Uses & $\mathrm{NC}$ & RI \\
\hline $\begin{array}{l}\text { Abarema jupunba (Willd.) } \\
\text { Britton \& Killip var. } \\
\text { Jupunba }\end{array}$ & Babatenom & $\mathrm{T}$ & NEN & $\mathrm{Ba}$ & $\begin{array}{l}\mathrm{Gf} / \mathrm{Dc} / \\
\text { If }\end{array}$ & $\begin{array}{c}\text { Polycystic ovary/Inflammation/ } \\
\text { Healing }\end{array}$ & 4 & 0.57 \\
\hline $\begin{array}{l}\text { Amburana cearensis } \\
\text { (Allemão) A. C.Sm. }\end{array}$ & Cumarú & $\mathrm{T}$ & NEN & $\mathrm{Ba} / \mathrm{Sd}$ & $\begin{array}{l}\mathrm{Dc} / \mathrm{Gf} / \\
\mathrm{Lb} / \mathrm{Po}\end{array}$ & $\begin{array}{l}\text { Inflamed tooth/Diabetes/ } \\
\text { Expectoration/Fever/Flu/Infection/ } \\
\text { Inside inflammation/Sinusitis/Cough }\end{array}$ & 20 & 1 \\
\hline $\begin{array}{l}\text { Anadenanthera colubrina } \\
\text { (Vell) Brenan }\end{array}$ & Angico & $\mathrm{T}$ & NEN & $\mathrm{Ba}$ & $\mathrm{Lb}$ & $\begin{array}{c}\text { Stomachache/Expectoration/Flu/ } \\
\text { Cough }\end{array}$ & 8 & 0.62 \\
\hline $\begin{array}{l}\text { Bauhinia cheilantha } \\
\text { (Bong.) Steud }\end{array}$ & Mororó & $\mathrm{T}$ & NEN & $\mathrm{Ba}$ & Dc & Sexual impotence & 1 & 0.19 \\
\hline $\begin{array}{l}\text { Bauhinia variegata } \\
\text { L. }(25.586)\end{array}$ & Pata de vaca & $\mathrm{T}$ & $\mathrm{EC}$ & Lf & Dc & Hypertension/Diabetes & 2 & 0.38 \\
\hline $\begin{array}{l}\text { Cenostigma pyramidale } \\
\text { (Tul.) E. Gagnon \& G. P. } \\
\text { Lewis (25.587) }\end{array}$ & Catingueira & $\mathrm{T}$ & $\mathrm{EN}$ & $\mathrm{Ba} / \mathrm{Fl}$ & $\begin{array}{l}\mathrm{Dc} / \mathrm{If} / \\
\mathrm{Mc}\end{array}$ & $\begin{array}{c}\text { Tummy ache (abdominal pain)/Flu/ } \\
\text { Hemorrhoids/Prostate pain }\end{array}$ & 6 & 0.76 \\
\hline $\begin{array}{l}\text { Centrosema brasilianum } \\
\text { (L.) Benth }\end{array}$ & Priquito & $\mathrm{L}$ & NEN & $\mathrm{Fl}$ & Dc & Flu/Cough & 2 & 0.38 \\
\hline $\begin{array}{l}\text { Erythrina velutina Willd } \\
(25.555)\end{array}$ & Mulungú & $\mathrm{T}$ & NEN & $\mathrm{Ib}$ & Dc & Memory loss & 1 & 0.19 \\
\hline Hymenaea courbaril L. & Jatobá & $\mathrm{T}$ & NEN & $\begin{array}{l}\mathrm{Ba} / \mathrm{Ib} / \\
\mathrm{Fr}\end{array}$ & $\begin{array}{l}\mathrm{Dc} / \mathrm{Gf} / \\
\mathrm{Nt} / \mathrm{Lb} / \\
\mathrm{Mc}\end{array}$ & $\begin{array}{l}\text { Pain/Stomachache/Expectoration/ } \\
\text { Flu/Hernia/Bruise/Rheumatism }\end{array}$ & 11 & 1.05 \\
\hline $\begin{array}{l}\text { Libidibia ferrea (Mart. ex } \\
\text { Tul.) L. P.Queiroz var. } \\
\text { ferrea (25.565) }\end{array}$ & Jucá & $\mathrm{T}$ & EN & Fr & $\mathrm{Dc} / \mathrm{Mc}$ & $\begin{array}{l}\text { Diabetes/"Open chest" (pain in the } \\
\text { sternum region) }\end{array}$ & 2 & 0.38 \\
\hline $\begin{array}{l}\text { Machaerium hirtum } \\
\text { (Vell.) Stellfeld }\end{array}$ & Espinho rei & $\mathrm{T}$ & NEN & $\mathrm{Ba}$ & Dc & Diarrhea & 2 & 0.19 \\
\hline $\begin{array}{l}\text { Mimosa sensitiva var. } \\
\text { malitiosa (Mar.) barneby }\end{array}$ & Malícia & $S$ & EN & Ro & Dc & Healing & 1 & 0.19 \\
\hline $\begin{array}{l}\text { Pterodon emarginatus } \\
\text { Vogel. }\end{array}$ & Sucupira branca & $\mathrm{T}$ & NEN & $\mathrm{Sd}$ & Mc & Backache/Rheumatism & 3 & 0.24 \\
\hline $\begin{array}{l}\text { Senna occidentalis (L.) } \\
\text { Link }(25.572)\end{array}$ & Mata pasto & $S$ & NEN & Lf & $\mathrm{Nt}$ & Lumb & 1 & 0.19 \\
\hline $\begin{array}{l}\text { Vigna unguiculata (L.) } \\
\text { Walp. }\end{array}$ & $\begin{array}{l}\text { Feijão verde/ } \\
\text { Cowpeas }\end{array}$ & $\mathrm{L}$ & EC & $\mathrm{Lf} / \mathrm{Fr}$ & $\mathrm{Dc} / \mathrm{If} / \mathrm{Jc}$ & Anticoagulant/Malaise & 3 & 0.38 \\
\hline $\begin{array}{l}\text { Fabaceae sp. } \\
\text { Geraniaceae }\end{array}$ & Unha de gato & * & * & Lf & Dc & Rheumatism & 1 & 0.19 \\
\hline $\begin{array}{l}\text { Pelargonium graveolens } \\
\text { L'Hér. ex aiton }(25.566) \\
\text { Illiciaceae }\end{array}$ & Malva rosa & $\mathrm{H}$ & $\mathrm{EC}$ & $\mathrm{Fl} / \mathrm{Lf}$ & $\begin{array}{l}\mathrm{Dc} / \mathrm{If} / \\
\mathrm{Lb}\end{array}$ & $\begin{array}{c}\text { Tammy ache/Fever/Flu/Sinusitis/ } \\
\text { Cough }\end{array}$ & 13 & 0.67 \\
\hline Illicium verum Hook.f. & Star anise & $\mathrm{T}$ & $\mathrm{EC}$ & $\mathrm{Sd} / \mathrm{Fr}$ & $\begin{array}{l}\text { If/Dcl } \\
\text { Gf }\end{array}$ & $\begin{array}{c}\text { Pain/Polycystic ovary/Infection/ } \\
\text { Expectoration }\end{array}$ & 5 & 0.62 \\
\hline $\begin{array}{l}\text { Iridaceae } \\
\text { Eleutherine bulbosa (Mill.) } \\
\text { Urb. } \\
\text { Lamiaceae }\end{array}$ & $\begin{array}{l}\text { Alho bravo/Wild } \\
\text { garlic }\end{array}$ & $\mathrm{H}$ & NEN & $\mathrm{Ei}$ & Dc & Cough/Flu/Expectoration & 3 & 0.38 \\
\hline $\begin{array}{l}\text { Aeollanthus suaveolens } \\
\text { Mart. ex Spreng (25.594) }\end{array}$ & Macassá & $\mathrm{H}$ & $\mathrm{EC}$ & $\mathrm{Lf} / \mathrm{Sd}$ & $\begin{array}{c}\mathrm{Dc} / \mathrm{Nt} / \\
\mathrm{Jc}\end{array}$ & $\begin{array}{l}\text { Stroke/Headache/Earache/Flu/ } \\
\text { Sinusitis/ } \\
\text { Amebiasis/Bloated tummy } \\
\text { (abdominal fullness)/Bronchitis/ } \\
\text { Tranquilizer/Stomach cramps/ }\end{array}$ & 10 & 0.81 \\
\hline $\begin{array}{l}\text { Mentha arvensis } \\
\text { L. }(25.578)\end{array}$ & $\begin{array}{l}\text { Hortelã miúda/ } \\
\text { Small mint }\end{array}$ & $\mathrm{H}$ & $\mathrm{EC}$ & Lf & $\begin{array}{l}\mathrm{Dc} / \mathrm{If} / \\
\mathrm{Lb} / \mathrm{Mc} / \\
\mathrm{Sc}\end{array}$ & $\begin{array}{l}\text { Tummy ache (abdominal pain)/ } \\
\text { Headache/Nausea/Expectoration/ } \\
\text { Fever/Gas/Flu/Hemorrhoids/ } \\
\text { Inflammation/Intestine/Malaise/ } \\
\text { Gallbladder stone/Kidney stone/ } \\
\text { Constipation/Cough/Verminosis }\end{array}$ & 46 & 1.71 \\
\hline Ocimum basilicum L. & Alfavaca & $\mathrm{H}$ & EC & Lf/Sd & $\mathrm{Dc} / \mathrm{Nt}$ & Sinusitis/Speck in the eye & 2 & 0.38 \\
\hline $\begin{array}{l}\text { Ocimum gratissimum } \\
\text { L. }(25.580)\end{array}$ & Louro & $S$ & ENT & Lf & $\mathrm{Dc} / \mathrm{If}$ & $\begin{array}{l}\text { Bloated tummy (abdominal fullness)/ } \\
\text { Indigestion }\end{array}$ & 5 & 0.19 \\
\hline
\end{tabular}


TABle 1: Continued.

\begin{tabular}{|c|c|c|c|c|c|c|c|c|}
\hline $\begin{array}{l}\text { Family/Scientific name/ } \\
\text { Voucher }\end{array}$ & $\begin{array}{l}\text { Local name/ } \\
\text { English name }\end{array}$ & $\begin{array}{l}\text { Life } \\
\text { form }\end{array}$ & $\begin{array}{l}\text { Origin and } \\
\text { endemism }\end{array}$ & $\begin{array}{l}\text { Parts } \\
\text { used }\end{array}$ & $\begin{array}{c}\text { Form of } \\
\text { use }\end{array}$ & Uses & $\mathrm{NC}$ & RI \\
\hline $\begin{array}{l}\text { Plectranthus amboinicus } \\
\text { (Lour.) Spreng (25.550) }\end{array}$ & $\begin{array}{l}\text { Hortelã grande/ } \\
\text { Mint }\end{array}$ & $\mathrm{H}$ & EC & Lf & $\begin{array}{l}\mathrm{Dc} / \mathrm{If} / \\
\mathrm{Nt} / \mathrm{Lb} / \\
\mathrm{Jc}\end{array}$ & $\begin{array}{l}\text { Lumb/Inflamed tooth/Intestinal } \\
\text { detoxification/Tummy ache } \\
\text { (abdominal pain)/Headache/Earache/ } \\
\text { Expectoration/Fever/Flu/Cough }\end{array}$ & 30 & 1.19 \\
\hline $\begin{array}{l}\text { Plectranthus barbatus } \\
\text { Andrews }\end{array}$ & Boldo & $\mathrm{H}$ & $\mathrm{EC}$ & Lf & $\mathrm{Dc} / \mathrm{If}$ & $\begin{array}{l}\text { Bloated tummy (abdominal fullness)/ } \\
\text { Indigestion/Tummy ache (abdominal } \\
\text { pain) }\end{array}$ & 9 & 0.29 \\
\hline Rosmarinus officinalis $\mathrm{L}$. & $\begin{array}{l}\text { Alecrim/ } \\
\text { Rosemary }\end{array}$ & $\mathrm{H}$ & EC & Lf & $\begin{array}{l}\mathrm{Dc} / \mathrm{If} / \\
\mathrm{Mc}\end{array}$ & $\begin{array}{c}\text { Accelerated Heart/Pain/ } \\
\text { Hypertension/Injury/Malaise/ } \\
\text { Nervousness }\end{array}$ & 7 & 0.71 \\
\hline Lauraceae & & & & & & & & \\
\hline $\begin{array}{l}\text { Cinnamomum zeylanicum } \\
\text { Blume }\end{array}$ & $\begin{array}{l}\text { Canela/ } \\
\text { Cinnamon }\end{array}$ & $\mathrm{T}$ & $\mathrm{EC}$ & $\mathrm{Ba}$ & If & Indigestion/Pain & 2 & 0.38 \\
\hline Persea americana Mill. & $\begin{array}{l}\text { Abacate/ } \\
\text { Avocado }\end{array}$ & $\mathrm{T}$ & ENT & $\mathrm{Sd} / \mathrm{Lf}$ & $\mathrm{Mc} / \mathrm{Dc}$ & Injury/Kidney inflammation & 2 & 0.38 \\
\hline Punica granatum L. & $\begin{array}{c}\text { Romã/ } \\
\text { Pomegranate }\end{array}$ & $\mathrm{T}$ & EC & Fr & $\mathrm{Dc} / \mathrm{Mc}$ & $\begin{array}{l}\text { Throat/Sore throat/Throat } \\
\text { inflammation/Throat infection/ } \\
\text { Infection/Inflammation/Hoarseness }\end{array}$ & 15 & 0.67 \\
\hline $\begin{array}{l}\text { Malvaceae } \\
\text { Abelmoschus esculentus } \\
\text { (L.) Moench (25.569) }\end{array}$ & Quiabo/Okra & $\mathrm{H}$ & $\mathrm{EC}$ & Fr & Mc & Diabetes & 1 & 0.19 \\
\hline $\begin{array}{l}\text { Ceiba glaziovii (Kuntze) } \\
\text { K. Schum. } \\
\text { Meliaceae }\end{array}$ & Barriguda & $\mathrm{T}$ & EN & $\mathrm{Ba}$ & Dc & "Barriga d'água" (Schistosomiasis) & 1 & 0.19 \\
\hline Cedrela odorata L. & Cedro & $\mathrm{T}$ & NEN & $\mathrm{Ba}$ & $\mathrm{Dc} / \mathrm{If}$ & $\begin{array}{l}\text { Bloated tummy (abdominal fullness)/ } \\
\text { Hatched burp (a burp smelling rotten } \\
\text { egg) }\end{array}$ & 4 & 0.19 \\
\hline $\begin{array}{l}\text { Moraceae } \\
\text { Maclura tinctoria (L.) } \\
\text { D. Don ex Steud } \\
\text { Musaceae }\end{array}$ & Tatajuba & $\mathrm{T}$ & NEN & Lt & $\mathrm{Nt}$ & Toothache & 1 & 0.19 \\
\hline $\begin{array}{l}\text { Musa paradisiaca L. } \\
\text { Myrtaceae }\end{array}$ & $\begin{array}{l}\text { Bananeira/ } \\
\text { Banana tree }\end{array}$ & $\mathrm{H}$ & $\mathrm{EC}$ & $\mathrm{Fl} / \mathrm{Lt}$ & $\mathrm{Nt} / \mathrm{Lb}$ & Gastritis/Cough & 3 & 0.38 \\
\hline Eucalyptus sp. & $\begin{array}{l}\text { Eucalipto/ } \\
\text { Eucalyptus }\end{array}$ & $\mathrm{T}$ & $\mathrm{EC}$ & Lf & $\begin{array}{l}\mathrm{Dc} / \mathrm{Nt} / \\
\mathrm{If} / \mathrm{Lb} / \\
\mathrm{Mc}\end{array}$ & $\begin{array}{l}\text { Tranquilizer/Fever/Throat/Flu/ } \\
\text { Infection/Sinusitis }\end{array}$ & 18 & 0.71 \\
\hline $\begin{array}{l}\text { Eugenia uniflora L } \\
(25.591)\end{array}$ & $\begin{array}{c}\text { Pitanga/Brazilian } \\
\text { cherry }\end{array}$ & $S$ & NEN & Lf & If & Tummy ache (abdominal pain) & 1 & 0.19 \\
\hline $\begin{array}{l}\text { Plinia cauliflora (Mart.) } \\
\text { Kausel }\end{array}$ & Jabuticaba & $\mathrm{T}$ & EN & $\begin{array}{c}\mathrm{Lf} / \mathrm{Fr} / \\
\mathrm{Ba}\end{array}$ & $\begin{array}{c}\text { If } / \mathrm{Nt} / \\
\mathrm{Mc}\end{array}$ & Diarrhea/Malaise & 3 & 0.24 \\
\hline Psidium guajava $\mathrm{L}$. & Goiaba/Guava & $\mathrm{T}$ & ENT & $\mathrm{Ba} / \mathrm{Lf}$ & $\begin{array}{c}\mathrm{Dc} / \mathrm{Nt} / \\
\mathrm{Mc}\end{array}$ & Dysentery/Tooth inflammation & 7 & 0.29 \\
\hline Psidium sp. & Cumati & $S$ & $\mathrm{~N} *$ & Lf & $\mathrm{Nt}$ & Tummy ache (abdominal pain) & 1 & 0.19 \\
\hline $\begin{array}{l}\text { Syzygium aromaticum (L.) } \\
\text { Nerril }\end{array}$ & $\begin{array}{l}\text { Cravo da India/ } \\
\text { Indian clove }\end{array}$ & $\mathrm{T}$ & $\mathrm{EC}$ & $\mathrm{Sd}$ & $\mathrm{Dc} / \mathrm{Gf}$ & Body pain/Polycystic ovary & 2 & 0.38 \\
\hline $\begin{array}{l}\text { Syzygium cumini (L.) } \\
\text { Skeels } \\
\text { Passifloraceae }\end{array}$ & Oliveira & $\mathrm{T}$ & ENT & $\mathrm{Fl}$ & Dc & Diabetes/Kidney stone & 2 & 0.38 \\
\hline $\begin{array}{l}\text { Passiflora edulis Sims } \\
\text { (25.576) }\end{array}$ & $\begin{array}{l}\text { Maracujá/ } \\
\text { Passion fruit }\end{array}$ & $\mathrm{L}$ & NEN & Fr & Po & Diabetes & 1 & 0.19 \\
\hline $\begin{array}{l}\text { Turnera subulata Sm. } \\
(25.548) \\
\text { Pedaliaceae }\end{array}$ & $\begin{array}{l}\text { Nove horas (Nine } \\
\text { o'clock) }\end{array}$ & $\mathrm{S}$ & NEN & $\mathrm{Fl}$ & If & Cough & 1 & 0.19 \\
\hline $\begin{array}{l}\text { Sesamum orientale L. } \\
\text { Piperaceae }\end{array}$ & Gergelim/Sesame & $\mathrm{H}$ & EC & Lf/Sd & $\mathrm{Nt} / \mathrm{Dc}$ & Conjunctivitis/Stroke & 2 & 0.38 \\
\hline $\begin{array}{l}\text { Piper nigrum L. } \\
\text { Phyllanthaceae }\end{array}$ & Pimenta/Pepper & $\mathrm{L}$ & $\mathrm{EC}$ & Lf & If $/ \mathrm{Nt}$ & Lumb/Thrombosed hemorrhoids & 2 & 0.38 \\
\hline
\end{tabular}


TABLE 1: Continued.

\begin{tabular}{|c|c|c|c|c|c|c|c|c|}
\hline $\begin{array}{l}\text { Family/Scientific name/ } \\
\text { Voucher }\end{array}$ & $\begin{array}{l}\text { Local name/ } \\
\text { English name }\end{array}$ & $\begin{array}{l}\text { Life } \\
\text { form }\end{array}$ & $\begin{array}{l}\text { Origin and } \\
\text { endemism }\end{array}$ & $\begin{array}{l}\text { Parts } \\
\text { used }\end{array}$ & $\begin{array}{c}\text { Form of } \\
\text { use }\end{array}$ & Uses & $\mathrm{NC}$ & RI \\
\hline $\begin{array}{l}\text { Phyllanthus niruri } \\
\text { L. (25.552) } \\
\text { Poaceae }\end{array}$ & $\begin{array}{l}\text { Quebra pedra/ } \\
\text { Stonebreaker }\end{array}$ & $\mathrm{H}$ & NEN & Ei/Ro & $\mathrm{Dc} / \mathrm{If}$ & Kidney stone/Kidneys & 5 & 0.24 \\
\hline $\begin{array}{l}\text { Cymbopogon citratus } \\
\text { (DC.) Stapf (25.556) }\end{array}$ & Capim santo & $\mathrm{H}$ & ENT & Lf & $\begin{array}{l}\mathrm{Dc} / \mathrm{If} / \\
\mathrm{Lb}\end{array}$ & $\begin{array}{l}\text { Bloated tummy (abdominal fullness)/ } \\
\text { Tranquilizer/Tiredness/Pain/Tummy } \\
\text { ache (abdominal pain)/Headache/ } \\
\text { Indigestion/Expectorant/Flu/Fever/ } \\
\text { Intestine/Nervousness/Blood } \\
\text { pressure/Sinusitis/Cough }\end{array}$ & 55 & 1.19 \\
\hline $\begin{array}{l}\text { Cynodon dactylon (L.) } \\
\text { Pers (25.589) }\end{array}$ & $\begin{array}{l}\text { Grama do rio/ } \\
\text { River grass }\end{array}$ & $\mathrm{H}$ & ENT & $\mathrm{Ei}$ & Dc & Sexual impotence & 1 & 0.19 \\
\hline $\begin{array}{l}\text { Zea mays L. (25.582) } \\
\text { Rhamnaceae }\end{array}$ & Milho/Corn & $\mathrm{H}$ & EC & $\mathrm{Sg}$ & Dc & Swelling & 2 & 0.19 \\
\hline Ziziphus joazeiro Mart. & Juá & $\mathrm{T}$ & EN & $\mathrm{Ba} / \mathrm{Lf}$ & $\begin{array}{c}\mathrm{Nt} / \mathrm{Lb} / \\
\mathrm{Mc}\end{array}$ & Expectoration/Flu/Seborrhea/Cough & 10 & 0.62 \\
\hline $\begin{array}{l}\text { Rubiaceae } \\
\text { Borreria verticillata (L.) } \\
\text { G. Mey }(25.554)\end{array}$ & $\begin{array}{l}\text { Vassoura de } \\
\text { botão }\end{array}$ & $S$ & NEN & Ro & $\mathrm{Dc} / \mathrm{Lb}$ & Lumb/Hemorrhoids/Flu & 3 & 0.57 \\
\hline $\begin{array}{l}\text { Genipa americana } \mathrm{L} \\
(25.564)\end{array}$ & Genipapo manso & $\mathrm{T}$ & NEN & Fr & $\mathrm{Mc}$ & Diabetes & 2 & 0.19 \\
\hline $\begin{array}{l}\text { Tocoyena bullata (Vell.) } \\
\text { Mart. ( } 25.581) \\
\text { Rutaceae }\end{array}$ & Genipapo bravo & $S$ & EN & $\mathrm{Ba}$ & $\mathrm{Dc} / \mathrm{Nt}$ & $\begin{array}{l}\text { Bruise/Dislocated finger or toe/Joint } \\
\text { pain }\end{array}$ & 3 & 0.57 \\
\hline $\begin{array}{l}\text { Citrus sinensis (L.) Osbeck } \\
(25.551)\end{array}$ & Laranja/Orange & $\mathrm{T}$ & $\mathrm{EC}$ & $\mathrm{Fl} / \mathrm{Lf}$ & $\begin{array}{l}\mathrm{Dc} / \mathrm{If} / \\
\mathrm{Lb}\end{array}$ & $\begin{array}{l}\text { Bloated tummy (abdominal fullness)/ } \\
\text { Tranquilizer/Dysentery/Headache/ } \\
\text { Fever/Flu/Insomnia/Sinusitis/Cough }\end{array}$ & 27 & 1 \\
\hline $\begin{array}{l}\text { Citrus aurantifolia } \\
\text { Swingle (25.584) }\end{array}$ & Limão/Lemon & $\mathrm{T}$ & EC & Fr & $\mathrm{Dc} / \mathrm{Jc}$ & Expectoration/Flu/Cough & 4 & 0.43 \\
\hline Ruta graveolens L. & Arruda/Rue & $\mathrm{H}$ & EC & $\mathrm{Fl}$ & $\begin{array}{l}\mathrm{Dc} / \mathrm{Nt} / \\
\mathrm{If} / \mathrm{Mc} / \\
\mathrm{Sm}\end{array}$ & $\begin{array}{c}\text { Stomach cramps/Pain/Toothache/ } \\
\text { Earache/Inflammation }\end{array}$ & 16 & 0.81 \\
\hline $\begin{array}{l}\text { Sapotaceae } \\
\text { Sideroxylon obtusifolium } \\
\text { (Roem. e Schult.) Penn. } \\
\text { Solanaceae }\end{array}$ & Quixabeira & $\mathrm{T}$ & NEN & $\mathrm{Ba}$ & Dc & Bruise & 1 & 0.19 \\
\hline $\begin{array}{l}\text { Solanum americanum } \\
\text { Mill (25.592) }\end{array}$ & Erva moura & $\mathrm{H}$ & NEN & Lf & If $/ J c$ & $\begin{array}{c}\text { Anticoagulant/Fracture/Injury/ } \\
\text { Bruise }\end{array}$ & 4 & 0.43 \\
\hline $\begin{array}{l}\text { Solanum melongena L. } \\
\text { Urticaceae }\end{array}$ & $\begin{array}{l}\text { Beringela/ } \\
\text { Eggplant }\end{array}$ & $\mathrm{H}$ & $\mathrm{EC}$ & Fr & $\mathrm{Mc}$ & Cholesterol & 2 & 0.19 \\
\hline $\begin{array}{l}\text { Cecropia pachystachya } \\
\text { Trécul } \\
\text { Verbenaceae }\end{array}$ & Capeira & $\mathrm{T}$ & NEN & Ro & Dc & Backache & 1 & 0.19 \\
\hline $\begin{array}{l}\text { Lippia alba (Mill.) N. E.Br. } \\
\text { ex P. Wilson (25.570) }\end{array}$ & Erva cidreira & $S$ & NEN & Lf/Ro & $\begin{array}{l}\mathrm{Dc} / \mathrm{If} / \\
\mathrm{Lb}\end{array}$ & $\begin{array}{c}\text { Anemia/“bad tummy” (abdominal } \\
\text { pain)/Bronchitis/Tranquilizer/ } \\
\text { Tiredness/Pain/Tummy ache } \\
\text { (abdominal pain)/Headache/Nausea/ } \\
\text { Expectoration/Fever/Flu/Intestine/ } \\
\text { Nerves/Cough }\end{array}$ & 51 & 1.24 \\
\hline $\begin{array}{l}\text { Zingiberaceae } \\
\text { Alpinia zerumbet (Pers.) }\end{array}$ & & & & & & & & \\
\hline $\begin{array}{l}\text { B. L. Burtt \& R. M. Sm. } \\
(25.559)\end{array}$ & Colônia & $\mathrm{H}$ & EC & $\mathrm{Fl} / \mathrm{Lf}$ & $\begin{array}{l}\text { Dc/If/ } \\
\text { Lb }\end{array}$ & Fever/Flu/Sinusitis/Cough & 15 & 0.48 \\
\hline Curcuma longa L. & Açafroa & $\mathrm{H}$ & EC & $\mathrm{Fl}$ & Dc & Jaundice & 1 & 0.19 \\
\hline $\begin{array}{l}\text { Zingiber officinale Roscoe. } \\
\text { Indeterminated }\end{array}$ & Gengibre/Ginger & $\mathrm{H}$ & EC & $\mathrm{Ri}$ & $\mathrm{Lb}$ & Bronchitis & 1 & 0.19 \\
\hline Indet. & Malva branca & $*$ & * & Ro & $\mathrm{Lb}$ & Flu & 1 & 0.19 \\
\hline
\end{tabular}

H: herbaceous; T: tree; S: shrub; L: liana; EN: endemic native; NEN: nonendemic native; $\mathrm{N}^{*}$ : native without data found about endemism; ENT: exotic naturalized; EC: exotic cultivated; Ei: entire plant; Bb: bulb; Bl: bulbils; Lf: leaf; Sd: seed; Fr: fruit; Fl: flower; Ba: bark; Ib: inner bark; Lt: latex; Tb: tubercle; St: stem; Ri: rhizome; Ef: stigmata; Ro: root; Sy: syrup; If: infusion; Mc: macerated; In: natural; Dc: decoction; Lb: "Lambedor"; Gf: "garrafada"; Sp: suppository; Jc: juice; As: ash; Po: powder; NC: number of citations; RI-Relative Importance. * Data on life form, origin, or endemism not found. 
without endemic data. Other species were exotic, being 41 cultivated and 12 naturalized (Table 1 ).

Herbaceous plants were most commonly used, similarly to other studies from Brazil $[27,58,62]$ as well as in other countries, such as Iraq [63], Ethiopia [64], Pakistan [65], Ecuador [66], Peru [67], and Bolivia [68]. These results are probably due to the fact that herbaceous plants have greater ease of cultivation or greater availability in areas close to the community. On the other hand, in a different Caatinga area in Brazil, a greater number of tree species were observed [60], similarly to Guinea-Bissau [34], probably due to climatic conditions not favorable to the development of herbaceous species in these areas.

The predominance of herbaceous plants can be explained by the hypothesis of appearance, from the biochemical perspective, which considers herbaceous plants as apparent by their greater production of chemical substances as a defense against herbivory [27].

The use of 36 trees, 19 shrubs, and 11 lianas species can also be considered representative when compared to data obtained from floristic and phytosociological studies in areas of Caatinga which mention 17 to 91 shrub-tree species or woody species [23, 24, 69-71]. Various studies on medicinal plants of the Caatinga have been published, and when only the woody species (shrubs and trees) and native species of this biome are observed, we found a similar number of species, with small variations. The present study recorded 66 medicinal woody species, compared to other studies in this biome that recorded 51 [25], 58 [20], and 37 [72]. This research evidences the vast array of woody species being recognized with medicinal potential in the northeastern semiarid region, requiring studies that more specifically evaluate its potential as a new drug.

A practically equivalent number of native species (56) was observed in relation to the number of exotic species (53). Some studies in Caatinga areas have recorded even a larger number of exotic species [73,74]. In these cases, a high number of exotic species can occur because these plants have greater availability and easier cultivation [73].

The hypothesis of diversification provides a possible explanation for the incorporation of exotic species into local pharmacopeias, considering that this insertion occurs through the need to expand the types of chemical components available for treatment [41].

A relatively high number of species was cultivated, similar to what was found in another semiarid area in Brazil [74]. In the Peruvian Amazon, native species of spontaneous occurrence predominated, which may have occurred due to the wide plant diversity and medicinal knowledge recorded in the study (303 species, of which 199 were native) [56]. The large number of cultivated species observed in the present study can be related to the herbaceous life form which makes cultivation easier in yards. Such life form also stood out in this research.

Another factor observed in the studied community that may be related to the predominance of cultivated species was the fact that there were almost no more native forest areas left close to the community since most of the area was converted into areas for agriculture and pasture. Other authors also present the urbanization process as responsible for the loss of forest areas and the consequent decrease or local extinction of native species [75]. Thus, medicinal plants grown in backyards, which are mostly herbaceous and exotic species, were an easier and viable way to obtain medicinal resources.

\subsection{Plant Parts, Methods of Preparation, Routes of Administration,} and Therapeutic Indications. Leaf was the most frequently used plant part (440 citations), bark (98), and flower and fruit (54 each) (Figure 2). Similar results were found in the state of Paraná, Brazil [62], Peru [56], Bolivia [68], and Madagascar [76]. In the semiarid region of Paraíba, Brazil, a greater use for bark was observed [25]. In Mexico [77], as observed among healers in Peru [78], the use of leaves and the whole plant was more common. The use of a given plant structure may vary depending on availability and user needs [79]. The high leaf use can be explained by the registration of a large number of herbaceous plants, being easier to collect, and there are many traditional medicines prepared with leaves, as well as the fact that the leaf has a higher concentration of metabolites [79]; on the other hand, the bark usually has a greater representation in studies developed in drier areas, where the leaves are not as available [80].

Regarding the preparation mode, there was an emphasis on decoction (291 citations), "Lambedor" (150), and infusion (122) (Figure 3). Decoction and infusion were also highlighted in another area of Agreste in the state of Paraíba, Brazil [17], as well as in Bolivia [68]. "Lambedor" is a local name attributed to a form of medicinal preparation, consisting of the combination and cooking of different barks and/or herbs, forming a kind of syrup, whereas "garrafada" is the maceration of barks, roots, or seeds (together) of different species, in water, cachaça, or white wine.

In the communities of the Italian Alps, the common preparations were infusion, maceration, and cataplasm [35], and in China, crushed material, decoction, and toasting [36].

The oral application was most common (662 citations) followed by topical (82) and inhalation (12). The most common route of administration was oral, which can also be observed in other studies $[3,66,67]$. The oral route may have a faster absorption of the chemicals compared to the other topical routes (topical and inhalation), and it is possible to observe that the topical route has an application more related to the lesions of the skin, whereas the inhalation route is more related to the respiratory system.

The most cited therapeutic indications were flu (116), cough (97), and dyspepsia (indigestion) (48), similar to the findings observed by other authors $[8,81,82]$. The prominence observed for these diseases is possible because these diseases affect the community in a more common way, being more frequent and widespread for the treatments for these diseases.

3.4. Most Mentioned and Most Versatile Species. The plants mentioned by the largest number of participants were D. ambrosioides (mentruz) (59 citations), C. citratus 


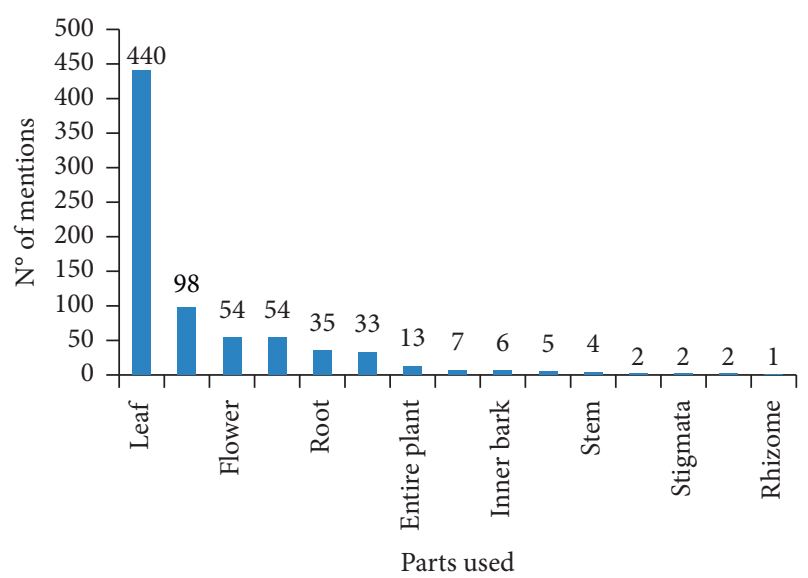

Figure 2: Mentions of plant parts known for medicinal use by residents of the Malícia community, Araçagi-PB (Northeastern Brazil).

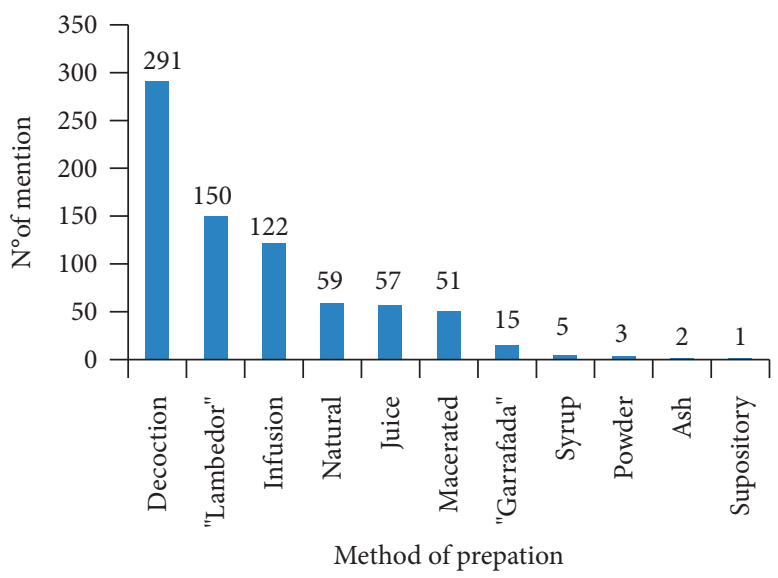

Figure 3: Mentions of methods of preparation of medicinal plants known by the residents of the Malícia community, Araçagi, Paraíba (Northeastern Brazil).

(capim santo) (55), and L. alba (51) (erva cidreira), which are all considered exotic in the semiarid region of Brazil, being more prominent for diseases of the respiratory and digestive systems, as well as parasitic diseases, in the case of D. ambrosioides.

D. ambrosioides is a species widely used in folk medicine and described in several studies on medicinal plants with different uses [83], and its antioxidant and anti-inflammatory bioactivity has been observed [84]. The uses mentioned for this species in the present work refer mainly to the treatment of parasitic diseases and the respiratory and digestive systems. The antioxidant bioactivity [84] of this plant might explain its efficacy in the treatment of gastric ulcers, one of the properties attributed to it by informants.

Pharmacological investigations for C. citratus found several bioactive effects of this plant [85]. Among these effects, we can highlight the antidiarrheal activity and sedative activity [85], which underline some of the indications given to this plant as diarrhea and nervousness.
L. alba was found to have antiviral and analgesic activity, which might explain the traditional uses indicated for this plant as influenza [86].

It was observed that 10 species presented high versatility with $\mathrm{RI} \geq 1$. The species with greater versatility were $M$. arvensis (Small mint) $(\mathrm{RI}=1.71)$, A. vera (Babosa) (1.62), and $M$. urundeuva (Aroeira) (1.52). In Caatinga areas, the highest values of RI were attributed to M. urundeuva and $S$. obtusifolium [25], similar to other areas of Caatinga in the state of Paraíba $[87,88]$. In different areas of Caatinga, in the Northeast of Brazil, M. urundeuva $(\mathrm{RI}=2)$, A. cearensis (2), and $M$. rigida $(1,9)$ [89] were the most versatile.

No correspondence was observed between the most cited species and the most versatile species, indicating that a larger number of citations will not necessarily imply greater versatility (diversity of uses) [51]. Although a species presents a high number of citations, its RI will not be high if these citations are concentrated in restricted groups of properties and bodily systems; in order to obtain a high RI, it is necessary to observe a great diversity of uses attributed to a species.

3.5. Categories and Informant Consensus. The most relevant uses were symptoms and signs not classified elsewhere (268 citations), diseases of the respiratory system (192), and diseases of the digestive system (116) (Figure 4). It is common that diseases of the respiratory and digestive systems are frequently treated by traditional medicine [4] as a result of covering the most common diseases that affect the population [90]. However, some differences could be observed in other studies, e.g., the prevalence of digestive system, reproductive system, and cardiovascular system disorders [91], and in the case of emphasis on the treatment of conditions of the skin and gastrointestinal and urogenital systems [92]. We believe that this may be a reflection of the environmental, cultural, economic, and social conditions of the studied regions.

Some studies suggest that the widespread use of medicinal plants to treat diseases of the respiratory system and digestive system may be due to local conditions such as air pollution, presence of impurities in water, and smoke caused by wood-burning, which make the population more prone to manifest these diseases [93]. For example, in the Araguaia microregion in the state of Mato Grosso, Brazil, a higher number of citations were observed for infectious and parasitic diseases, followed by digestive and respiratory system diseases, which may be due to the sanitation conditions recorded in the study [54].

The Informant Consensus Factor (ICF) identified a strong consensus $(>0.50)$ for seven categories, with a greater emphasis on neoplasms $(\mathrm{ICF}=1)$, nervous system diseases $(0,83)$, and infectious and parasitic diseases $(0,82)$ (Table 2$)$. In a region of Mexico, a higher ICF was observed for diseases of the respiratory system (0.92), diseases of the digestive system (0.91), and infections and parasitic diseases (0.89) [77], compared, e.g., to the Italian Alps respiratory (0.88), digestive (0.86), and integumentary (0.83) [35]. It does not seem to be common for the category of neoplasms to present 


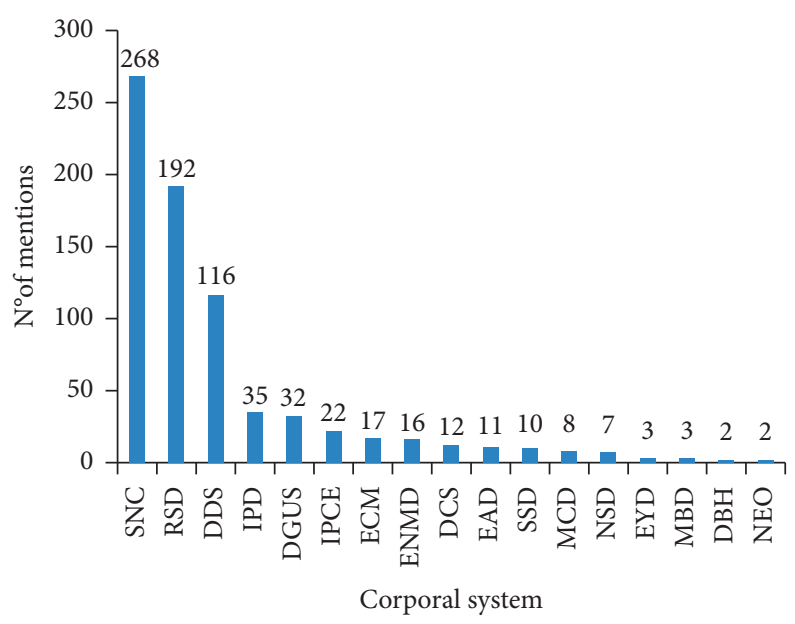

FIGURE 4: Body system mentions. ECM = external causes of morbidity and mortality; SSD = skin and subcutaneous tissue disorders; EAD = ear diseases; $\mathrm{DBH}=$ diseases of the blood and hematopoietic organs; DSC = diseases of the cardiovascular system; DDS = diseases of the digestive system; DGUS = diseases of the genito-urinary system; NSD = nervous system disorders; $\mathrm{MCD}=$ musculoskeletal and connective tissue diseases; RSD = respiratory system diseases; EYD = eye diseases; ENMD = endocrine, nutritional and metabolic diseases; IPD = infectious and parasitic diseases; IPCE = injuries, poisoning and some other consequences of external causes; NEO = neoplasms; SNC = symptoms and signs not elsewhere classified; $\mathrm{MBD}=$ mental and behavioral disorders.

TABLE 2: Informant consensus factor (ICF) for the categories of medicinal use cited.

\begin{tabular}{|c|c|c|c|}
\hline Corporal system & No. of species & No. of uses mentioned & ICF \\
\hline External causes of morbidity and mortality (ECM) & 10 & 17 & 0,44 \\
\hline Skin and subcutaneous tissue disorders (SSD) & 6 & 10 & 0,44 \\
\hline Ear diseases $(\mathrm{EAD})$ & 3 & 11 & 0,80 \\
\hline Diseases of the blood and hematopoietic organs (DBH) & 2 & 2 & - \\
\hline Diseases of the cardiovascular system (DCS) & 10 & 12 & 0,18 \\
\hline Diseases of the digestive system (DDS) & 38 & 116 & 0,68 \\
\hline Diseases of the genitourinary system (DGUS) & 15 & 32 & 0,55 \\
\hline Nervous system disorders (NSD) & 2 & 7 & 0,83 \\
\hline Musculoskeletal and connective tissue diseases (MCD) & 6 & 8 & 0,29 \\
\hline Respiratory system diseases (DSR) & 41 & 192 & 0,79 \\
\hline Eye diseases (EYD) & 3 & 3 & - \\
\hline Endocrine, nutritional and metabolic diseases (ENMD) & 14 & 16 & 0,13 \\
\hline Infectious and parasitic diseases (IPD) & 7 & 35 & 0,82 \\
\hline Injuries, poisoning and some other consequences of external causes (IPCE) & 16 & 22 & 0,29 \\
\hline Neoplasms (NEO) & 1 & 2 & 1,00 \\
\hline Symptoms and signs not elsewhere classified (SNC) & 56 & 268 & 0,79 \\
\hline Mental and behavioral disorders (MBD) & 3 & 3 & - \\
\hline
\end{tabular}

such a high ICF; the explanation for this might be given by the fact that there was only one plant mentioned in two citations for this category.

\section{Conclusions}

The data obtained indicate that the inhabitants of the Malícia community have a broad knowledge of a great diversity of medicinal plants, with different therapeutic applications.

The slightly higher use of native species than exotic species shows that although there has been a significant loss of native forest in the region, the local population maintains the practice and the knowledge of native plants, growing or even buying, in some cases, native plants unavailable in the region. However, a high number of uses of exotic species were also recorded, which may indicate a possibility of proving the hypothesis of diversification, that is, to analyze if the introduction of exotic species into the local pharmacopeia occurs as a possibility of treatment of a wider set of diseases, and it would be worth to investigate what other factors may be interfering with the dynamics of local knowledge. This might lead to reduced pressure on native species. It would also be interesting to conduct further studies in pharmacology and toxicology to confirm plant efficacy, as well as to identify if there is any toxicity in the mentioned plants.

\section{Data Availability}

The data used for this study are deposited in the database of the Laboratory of Ethnobiology and Environmental Sciences, of the Federal University of Paraíba, João Pessoa, 
Brazil, and can be accessed through the previous contact with the authors of this study.

\section{Conflicts of Interest}

The authors declare that they have no conflicts of interest.

\section{References}

[1] M. A. M. Maciel, A. C. Pinto, V. F. Veiga, N. F. Grynberg, and A. Echevarria, "Plantas medicinais: a necessidade de estudos multidisciplinares," Química Nova, vol. 25, no. 3, pp. 429-438, 2002.

[2] World Health Organization, Legal Status of Traditional Medicine and Complementary/Alternative Medicine: A Worldwide Review, World Health Organization, Geneva, Switzerland, 2001.

[3] M. C. d. M. Amorozo, "Uso e diversidade de plantas medicinais em Santo Antonio do Leverger, MT, Brasil," Acta Botanica Brasilica, vol. 16, no. 2, pp. 189-203, 2002.

[4] E. D. P. P. Pinto, M. C. d. M. Amorozo, and A. Furlan, "Conhecimento popular sobre plantas medicinais em comunidades rurais de mata atlântica - Itacaré, BA, Brasil," Acta Botanica Brasilica, vol. 20, no. 4, pp. 751-762, 2006.

[5] T. O. Issa, Y. S. Mohamed, S. Yagi et al., "Ethnobotanical investigation on medicinal plants in Algoz area (South Kordofan), Sudan," Journal of Ethnobiology and Ethnomedicine, vol. 14, no. 1, p. 31, 2018.

[6] F. Corroto, J. Rascón, E. Barboza, and M. J. Macía, "Medicinal plants for rich people vs. Medicinal plants for poor people: a case study from the Peruvian andes," Plants, vol. 10, no. 8, 2021.

[7] I. Vandebroek, V. Reyes-García, U. P. Albuquerque, R. Bussmann, and A. Pieroni, "Local knowledge: who cares?" Journal of Ethnobiology and Ethnomedicine, vol. 7, no. 35, 2011.

[8] J. A. Bhat, M. Kumar, and R. W. Bussmann, "Ecological status and traditional knowledge of medicinal plants in kedarnath wildlife sanctuary of garhwal himalaya, India," Journal of Ethnobiology and Ethnomedicine, vol. 9, no. 1, p. 1, 2013.

[9] U. P. D. Albuquerque and N. Hanazaki, "As pesquisas etnodirigidas na descoberta de novos fármacos de interesse médico e farmacêutico: fragilidades e pespectivas," Revista Brasileira de Farmacognosia, vol. 16, pp. 678-689, 2006.

[10] M. O. Faruque, S. B. Uddin, J. W. Barlow et al., "Quantitative ethnobotany of medicinal plants used by indigenous communities in the Bandarban district of Bangladesh," Frontiers in Pharmacology, vol. 9, p. 40, 2018.

[11] O. Kayser, "Ethnobotany and medicinal plant biotechnology: from tradition to modern aspects of drug development," Planta Medica, vol. 84, pp. 834-838, 2018.

[12] A. Boudjelal, C. Henchiri, M. Sari et al., "Herbalists and wild medicinal plants in M'Sila (North Algeria): an ethnopharmacology survey," Journal of Ethnopharmacology, vol. 148, no. 2, pp. 395-402, 2013.

[13] S. Aswani, A. Lemahieu, and W. H. H. Sauer, "Global trends of local ecological knowledge and future implications," PLoS One, vol. 23, 2018.

[14] M. F. Agra, G. S. Baracho, and I. J. D. Basílio, "Sinopse da Flora Medicinal do Cariri Paraibano," Oecologia Bras.vol. 11, no. 3, pp. 323-330, 2007.

[15] L. Beltreschi, R. B. de Lima, and D. D. da Cruz, "Traditional botanical knowledge of medicinal plants in a "quilombola" community in the Atlantic Forest of northeastern Brazil,"
Environment, Development and Sustainability, vol. 21, no. 3, pp. 1185-1203, 2019.

[16] R. F. P. Lucena, T. C. Soares, and C. F. A. Vasconcelos Neto, "Uso de recursos vegetais da Caatinga em uma comunidade rural no Curimataú paraibano (Nordeste do Brasil)," Polibotánica, vol. 34, pp. 217-238, 2012.

[17] J. M. P. Cordeiro and L. P. Félix, "Conhecimento botânico medicinal sobre espécies vegetais nativas da caatinga e plantas espontâneas no agreste da paraíba, Brasil," Revista Brasileira de Plantas Medicinais, vol. 16, no. 3, pp. 685-692, 2014.

[18] C. M. Lucena, T. K. N. Carvalho, and E. A. Marín, "Potencial medicinal de cactáceas en la región semiárida del Nordeste de Brasil," Gaia Science Pte Ltd, vol. 23, pp. 36-50, 2014.

[19] M. F. M. Brito, R. F. P. Lucena, and D. D. Cruz, "Conhecimento etnobotânico local sobre plantas medicinais: uma avaliação de índices quantitativos," Interciencia, vol. 40, no. 3, pp. 156-164, 2015.

[20] N. A. Marreiros, E. C. Ferreira, C. M. Lucena, and R. F. P. Lucena, "Conhecimento botânico tradicional sobre plantas medicinais no semiárido da paraíba (Nordeste , Brasil)," Revista Ouricuri, vol. 5, no. 1, pp. 110-144, 2015.

[21] F. S. Medeiros, G. B. D. Sá, M. K. L. Dantas, and M. D. G. V. M. D. Almeida, "Plantas medicinais comercializadas na feira livre do município de Patos, Paraíba," Revista Verde de Agroecologia e Desenvolvimento Sustentável, vol. 14, no. 1, pp. 150-155, 2019.

[22] I. M. Pereira, L. A. d. Andrade, M. R. d. V. Barbosa, and E. V. S. B. Sampaio, "Composição florística e análise fitossociológica do componente arbustivo-arbóreo de um remanescente florestal no Agreste Paraibano," Acta Botanica Brasilica, vol. 16, no. 3, pp. 357-369, 2002.

[23] D. M. B. M. Trovão, A. M. Freire, and J. I. M. Melo, "Florística e fitossociologia do componente lenhoso da mata ciliar do riacho de Bodocongó, Semiárido paraibano," Revista Caatinga - SciELO, vol. 23, no. 2, pp. 78-86, 2010.

[24] A. V. Lacerda, F. M. Barbosa, J. J. Soares, and M. R. V. Barbosa, "Flora arbustiva-arbórea de três áreas ribeirinhas no semiárido paraibano, Brasil," Biota Neotropica, vol. 10, no. 4, pp. 277-284, 2010.

[25] P. C. Coutinho, Z. A. Soares, and E. C. Ferreira, "Knowledge and use of medicinal plants in the Semiarid Region of Brazil," Brazilian Journal of Biology, vol. 2, no. 3, pp. 51-74, 2015.

[26] T. C. Silva, J. M. Silva, and M. A. Ramos, "What factors guide the selection of medicinal plants in a local pharmacopoeia? A case study in a rural community from a historically transformed atlantic forest landscape," Evidence-based Complementary and Alternative Medicine, vol. 2018, Article ID 2519212, 11 pages, 2018.

[27] A. Lozano, E. L. Araújo, M. F. Medeiros, and U. P. Albuquerque, "The apparency hypothesis applied to a local pharmacopoeia in the Brazilian northeast," Journal of Ethnobiology and Ethnomedicine, vol. 10, no. 2, p. 2, 2014.

[28] G. T. Soldati and U. P. Albuquerque, "Ethnobotany in intermedical spaces: the case of the Fulni-ô Indians (Northeastern Brazil)," Evidence-based Complementary and Alternative Medicine, vol. 2012, Article ID 648469, 9 pages, 2012.

[29] J. H. A. Carlotto, J. Rodrigues, and A. Benites, "Notes on current Mbyá-Guarani medicinal plant exchanges in southern Brazil," Journal of Ethnobiology and Ethnomedicine, vol. 17, no. 38, 2021.

[30] N. C. B. Silva, A. C. D. Regis, M. A. Esquibel, J. E. S. Santos, and M. Z. Almeida, "Uso de plantas medicinais na comunidade quilombola da Barra II - Bahia , Brasil,” Boletín 
Latinoam. y del Caribe Plantas Med. y Aromáticas, vol. 11, no. 5, pp. 435-453, 2012.

[31] P. B. Yazbek, P. Matta, L. F. Passero et al., "Plants utilized as medicines by residents of quilombo da fazenda, núcleo picinguaba, ubatuba, são paulo, Brazil: a participatory survey," Journal of Ethnopharmacology, vol. 244, Article ID 112123, 2019.

[32] S. P. F. Vásquez, M. S. Mendonça, and S. N. Noda, "Etnobotânica de plantas medicinais em comunidades ribeirinhas do Município de Manacapuru, Amazonas, Brasil," Acta Amazonica, vol. 44, no. 4, pp. 457-472, 2014.

[33] M. R. D. Brito and L. D. Senna-Valle, "Plantas medicinais utilizadas na comunidade caiçara da Praia do Sono, Paraty, Rio de Janeiro, Brasil," Acta Botanica Brasilica, vol. 25, no. 2, pp. 363-372, 2011.

[34] L. Catarino, P. J. Havik, and M. M. Romeiras, "Medicinal plants of Guinea-Bissau: therapeutic applications, ethnic diversity and knowledge transfer," Journal of Ethnopharmacology, vol. 183, pp. 71-94, 2016.

[35] S. Vitalini, M. Iriti, C. Puricelli, D. Ciuchi, A. Segale, and G. Fico, "Traditional knowledge on medicinal and food plants used in Val San Giacomo (Sondrio, Italy)-An alpine ethnobotanical study," Journal of Ethnopharmacology, vol. 145, no. 2, pp. 517-529, 2013.

[36] X.-L. Zheng, J.-H. Wei, W. Sun, R.-T. Li, S.-B. Liu, and H.-F. Dai, "Ethnobotanical study on medicinal plants around Limu Mountains of hainan Island, China," Journal of Ethnopharmacology, vol. 148, no. 3, pp. 964-974, 2013.

[37] M. A. M. Alejandro, L. M. G. Campillo, and R. M. Méndez, "El uso de las plantas medicinales en las comunidades mayachontales de Nacajuca, Tabasco, México," Polibotánica, vol. 29, pp. 213-262, 2010.

[38] M. E. Acosta, N. D. Vignale, and A. H. Ladio, "Qué saben sobre plantas empleadas en medicina tradicional los niños de una escuela primaria de S. S. De jujuy, Argentina?" Gaia Science, vol. 9, no. 3, pp. 90-104, 2015.

[39] F. R. Santoro, W. S. Ferreira Júnior, T. A. S. Araújo, A. H. Ladio, and U. P. Albuquerque, "Does plant species richness guarantee the resilience of local medical systems? A perspective from utilitarian redundancy," PLoS One, vol. 10, no. 3, pp. 1-18, 2015.

[40] P. M. Medeiros, W. S. Ferreira Júnior, and F. S. Queiroz, "Utilitarian redundancy in local medical systems - theoretical and methodological contributions," Journal of Ethnobiology and Ethnomedicine, vol. 16, no. 62, 2020.

[41] N. Leal Alencar, T. A. de Sousa Araújo, E. L. C. de Amorim, and U. P. de Albuquerque, "The inclusion and selection of medicinal plants in traditional pharmacopoeias-evidence in support of the diversification hypothesis," Economic Botany, vol. 64 , no. 1 , pp. $68-79,2010$.

[42] R. F. P. D. Lucena, P. M. D. Medeiros, E. D. L. Araújo, A. G. C. Alves, and U. P. D. Albuquerque, "The ecological apparency hypothesis and the importance of useful plants in rural communities from Northeastern Brazil: an assessment based on use value," Journal of Environmental Management, vol. 96, no. 1, pp. 106-115, 2012.

[43] J. P. D. O. Ribeiro, T. K. N. Carvalho, J. E. D. S. Ribeiro et al., "Can ecological apparency explain the use of plant species in the semi-arid depression of Northeastern Brazil?" Acta Botanica Brasilica, vol. 28, no. 3, pp. 476-483, 2014.

[44] N. M. Guerra, T. K. N. Carvalho, J. E. D. S. Ribeiro et al., "Ecological apparency hypothesis and plant utility in the semiarid region of Brazil," Ethnobotany Research and Applications, vol. 14, pp. 423-435, 2015.
[45] J. R. F. Lima, C. A. B. Alves, and J. E. S. Ribeiro, "Uso e disponibilidade de espécies vegetais nativas no semiárido do nordeste do brasil: uma análise da hipótese da aparência ecológica," REDE - Rev. Eletrônica do PRODEMA, vol. 10, no. 1, pp. 110-131, 2016.

[46] R. C. L. Oliveira, E. M. F. Lins Neto, U. P. Albuquerque, and E. L. Araújo, "Prioridades de conservação de plantas medicinais," in Tópicos em Conservação, Etnobotânica e Etnofarmacologia de Plantas Medicinais e Mágicas, U. P. Albuquerque, C. F. C. B. R. Almeida and J. F. A. Marins, Eds., pp. 165-182, Springer, Berlin, Germany, 2005.

[47] IBGE | Portal do IBGE 2010. [Online]. Available: https://www. ibge.gov.br/.

[48] CPRM - Serviço Geológico do Brasil, "Projeto cadastro de fontes de abastecimento por água subterrânea. Diagnóstico do município de Aracagí, estado da Paraíba. Recife: CPRM/ PRODEEM," 2005.

[49] U. P. Albuquerque, R. F. P. Lucena, and N. L. Alencar, "Métodos e técnicas para coleta de dados etnobiológicos," in Métodos e técnicas na pesquisa etnobiológica e etnoecológica, R. F. P. Lucena and L. V. F. C. Cunha, Eds., pp. 39-64, 2010.

[50] ICD, "ICD-10 Version:2010," 2010. [Online]. Available: http://apps.who.int/classifications/icd10/browse/2010/en.

[51] B. C. Bennett and G. T. Prance, "Introduced plants in the indigenous pharmacopoeia of northern south America," Economic Botany, vol. 54, pp. 90-102, 1994.

[52] R. T. Trotter and M. H. Logan, "Informant consensus: a new approach for identifying potentially effective medicinal plants," in Plants In Indigenous Medicine and Diet, N. Etkin, Ed., Redgrave Publishing Company, New York, NY, USA, 1986.

[53] J. D. A. Silva, M. G. P. Nascimento, L. G. Grazina, K. N. C. Castro, S. J. Mayo, and I. M. Andrade, "Ethnobotanical survey of medicinal plants used by the community of Sobradinho, Lus Correia, Piau, Brazil," Journal of Medicinal Plants Research, vol. 9, no. 32, pp. 872-883, 2015.

[54] R. V. Ribeiro, I. G. C. Bieski, S. O. Balogun, and D. T. d. O. Martins, "Ethnobotanical study of medicinal plants used by ribeirinhos in the north Araguaia microregion, Mato Grosso, Brazil," Journal of Ethnopharmacology, vol. 205, pp. 69-102, 2017.

[55] B. F. de Santana, R. A. Voeks, and L. S. Funch, "Ethnomedicinal survey of a maroon community in Brazil's Atlantic tropical forest," Journal of Ethnopharmacology, vol. 181, pp. 37-49, 2016.

[56] G. Odonne, C. Valadeau, J. Alban-Castillo, D. Stien, M. Sauvain, and G. Bourdy, "Medical ethnobotany of the chayahuita of the paranapura basin (Peruvian Amazon)," Journal of Ethnopharmacology, vol. 146, no. 1, pp. 127-153, 2013.

[57] M. R. Zucchi, V. F. Oliveira Júnior, M. A. Gussoni, M. B. Silva, F. C. Silva, and N. E. Marques, "Levantamento etnobotânico de plantas medicinais na cidade de Ipameri - go," Revista Brasileira de Plantas Medicinais, vol. 15, no. 2, pp. 273-279, 2013.

[58] M. Giraldi and N. Hanazaki, "Uso e conhecimento tradicional de plantas medicinais no Sertão do Ribeirão, Florianópolis, SC, Brasil," Acta Botanica Brasilica, vol. 24, no. 2, pp. 395-406, 2010.

[59] M. F. Agra, G. S. Baracho, K. Nurit, I. J. L. D. Basílio, and V. P. M. Coelho, "Medicinal and poisonous diversity of the flora of "Cariri Paraibano," Brazil," Journal of Ethnopharmacology, vol. 111, no. 2, pp. 383-395, 2007.

[60] T. B. Gomes and F. P. S. D. F. Bandeira, "Uso e diversidade de plantas medicinais em uma comunidade quilombola no Raso 
da Catarina, Bahia," Acta Botanica Brasilica, vol. 26, no. 4, pp. 796-809, 2012.

[61] E. R. Oliveira and L. Menini Neto, "Levantamento etnobotânico de plantas medicinais utilizadas pelos moradores do povoado de Manejo, Lima Duarte - mg," Revista Brasileira de Plantas Medicinais, vol. 14, no. 2, pp. 311-320, 2012.

[62] M. Bolson, S. R. Hefler, E. I. Dall'Oglio Chaves, A. Gasparotto Junior, and E. L. Cardozo Junior, "Ethno-medicinal study of plants used for treatment of human ailments, with residents of the surrounding region of forest fragments of Paraná, Brazil," Journal of Ethnopharmacology, vol. 161, pp. 1-10, 2015.

[63] H. M. Ahmed, "Ethnopharmacobotanical study on the medicinal plants used by herbalists in Sulaymaniyah Province, Kurdistan, Iraq," Journal of Ethnobiology and Ethnomedicine, vol. 12, no. 1, p. 8, 2016.

[64] Z. Kassa, Z. Asfaw, and S. Demissew, "An ethnobotanical study of medicinal plants in sheka zone of southern nations nationalities and peoples regional state, Ethiopia," Journal of Ethnobiology and Ethnomedicine, vol. 16, no. 1, p. 7, 2020.

[65] H. Gulzar, A. Hazrat, and K. Gulzar, "Medicinal plants and their traditional uses in thana village, district malakand, khyber pakhtunkhwa, Pakistan," International Journal of Endorsing Health Science Research, vol. 7, no. 1, pp. 11-21, 2019.

[66] J. M. Andrade, H. Lucero Mosquera, and C. Armijos, "Ethnobotany of indigenous saraguros: medicinal plants used by community healers 'hampiyachakkuna' in the san lucas parish, southern Ecuador," BioMed Research International, vol. 2017, Article ID 9343724, 2017.

[67] M. Monigatti, R. W. Bussmann, and C. S. Weckerle, "Medicinal plant use in two Andean communities located at different altitudes in the Bolívar Province, Peru," Journal of Ethnopharmacology, vol. 145, no. 2, pp. 450-464, 2013.

[68] R. Quiroga, L. Meneses, and R. W. Bussmann, "Medicinal ethnobotany in huacareta (chuquisaca, Bolivia)," Journal of Ethnobiology and Ethnomedicine, vol. 8, no. 1, pp. 29-14, 2012.

[69] U. P. Albuquerque, L. H. C. Andrade, and J. Caballero, "Structure and floristics of homegardens in Northeastern Brazil," Journal of Arid Environments, vol. 62, no. 3, pp. 491-506, 2005.

[70] U. P. de Albuquerque, G. T. Soldati, S. S. Sieber, P. M. de Medeiros, J. C. de Sá, and L. C. de Souza, "Rapid ethnobotanical diagnosis of the Fulni-ô Indigenous lands (NE Brazil): floristic survey and local conservation priorities for medicinal plants," Environment, Development and Sustainability, vol. 13, no. 2, pp. 277-292, 2011.

[71] H. C. Silva, R. L. Caraciolo, L. C. Marangon, M. A. Ramos, L. L. Santos, and U. P. Albuquerque, "Evaluating different methods used in ethnobotanical and ecological studies to record plant biodiversity," Journal of Ethnobiology and Ethnomedicine, vol. 10, no. 48, p. 48, 2014.

[72] A. D. S. Souza, A. P. B. D. Souza, and R. F. P. D. Lucena, "Relative importance of medicinal plants in the semi-arid region of paraíba: a case study in the municipality of Congo (paraíba, northeast Brazil)," Brazilian Journal of Biological Sciences, vol. 3, no. 5, pp. 83-96, 2016.

[73] D. A. Ribeiro, D. G. Macêdo, L. G. S. Oliveira et al., "Potencial terapêutico e uso de plantas medicinais em uma área de Caatinga no estado do Ceará, nordeste do Brasil," Revista Brasileira de Plantas Medicinais, vol. 16, no. 4, pp. 912-930, 2014.

[74] S. L. Cartaxo, M. M. de Almeida Souza, and U. P. de Albuquerque, "Medicinal plants with bioprospecting potential used in semi-arid northeastern Brazil," Journal of Ethnopharmacology, vol. 131, no. 2, pp. 326-342, 2010.
[75] M. Shuaib, K. Ali, S. Ahmed et al., "Impact of rapid urbanization on the floral diversity and agriculture land of district Dir, Pakistan," Acta Ecologica Sinica, vol. 38, no. 6, pp. 394-400, 2018.

[76] A. D. Rabearivony, A. R. Kuhlman, Z. L. Razafiariso et al., "Ethnobotanical study of the medicinal plants known by men in Ambalabe, Madagascar," Ethnobotany Research and Applications, vol. 14, pp. 123-138, 2015.

[77] M. D. C. Juárez-Vázquez, C. Carranza-Álvarez, and A. J. Alonso-Castro, "Ethnobotany of medicinal plants used in Xalpatlahuac, Guerrero, México," Journal of Ethnopharmacology, vol. 148, no. 2, pp. 521-527, 2013.

[78] R. W. Bussmann and D. Sharon, "Traditional medicinal plant use in Loja province, Southern Ecuador," Journal of Ethnobiology and Ethnomedicine, vol. 2, no. 1, p. 44, 2006.

[79] S. Bahadur, M. S. Khan, M. Shah et al., "Traditional usage of medicinal plants among the local communities of Peshawar valley, Pakistan," Acta Ecologica Sinica, vol. 40, no. 1, pp. 1-29, 2020.

[80] U. P. Albuquerque, "Re-examining hypotheses concerning the use and knowledge of medicinal plants: a study in the Caatinga vegetation of NE Brazil," Journal of Ethnobiology and Ethnomedicine, vol. 2, 2006.

[81] S. Silva, M. G. V. Anselmo, and W. M. Dantas, "Conhecimento e uso de plantas medicinais em uma comunidade rural no município de Cuitegi, Paraíba, Nordeste do Brasil," Gaia Science, vol. 8, no. 1, pp. 249-267, 2014.

[82] J. A. Castro, B. P. Brasileiro, and D. H. Lyra, "Ethnobotanical study of traditional uses of medicinal plants: the flora of caatinga in the community of Cravolandia-BA, Brazil," Journal of Medicinal Plants Research, vol. 5, no. 10, pp. 1905-1917, 2011.

[83] A. P. Rodrigues and L. H. C. Andrade, "Levantamento etnobotânico das plantas medicinais utilizadas pela comunidade de inhamã, Pernambuco, Nordeste do brasil," Revista Brasileira de Plantas Medicinais, vol. 16, no. 3, pp. 721-730, 2014.

[84] K. Song, J. Zhang, P. Zhang et al., "Five new bioactive compounds fromChenopodium ambrosioides," Journal of Asian Natural Products Research, vol. 17, no. 5, pp. 482-490, May 2015.

[85] G. Shah, R. Shri, V. Panchal, N. Sharma, B. Singh, and A. Mann, "Scientific basis for the therapeutic use of Cymbopogon citratus, stapf (Lemon grass)," Journal of Advanced Pharmaceutical Technology \& Research, vol. 2, no. 1, pp. 3-8, 2011.

[86] T. Hennebelle, S. Sahpaz, H. Joseph, and F. Bailleul, "Ethnopharmacology of lippia alba," Journal of Ethnopharmacology, vol. 116, no. 2, pp. 211-222, 2008.

[87] R. F. P. Lucena, D. C. Farias, and T. K. N. Carvalho, "Uso e conhecimento da aroeira (Myracrodruon urundeuva) por comunidades tradicionais no Semiárido brasileiro," Sitientibus série Ciências Biológicas, vol. 11, no. 2, pp. 255-264, 2011.

[88] K. M. Pedrosa, D. S. Gomes, and C. M. Lucena, "Uso e disponibilidade local de Sideroxylon obtusifolium (Roem. \& Schult.) T.D. Penn. (Quixabeira) em três regiões da depressão sertaneja da Paraíba, Nordeste do Brasil,” Biofar, vol. 23, pp. 158-183, 2012.

[89] U. P. De Albuquerque, P. M. De Medeiros, A. L. S. De Almeida et al., "Medicinal plants of the caatinga (semi-arid) vegetation of NE Brazil: a quantitative approach," Journal of Ethnopharmacology, vol. 114, no. 3, pp. 325-354, 2007.

[90] C. F. C. B. R. Almeida and U. P. Albuquerque, "Uso e conservação de plantas e animais medicinais no estado de 
pernambuco (nordeste do brasil): um estudo de caso," Interciencia, vol. 27, no. 6, pp. 276-285, 2002.

[91] M. Razafindraibe, A. R. Kuhlman, H. Rabarison et al., "Medicinal plants used by women from Agnalazaha littoral forest (Southeastern Madagascar)," Journal of Ethnobiology and Ethnomedicine, vol. 9, no. 1, p. 73, 2013.

[92] A. Pieroni, A. Nedelcheva, A. Hajdari et al., "Local knowledge on plants and domestic remedies in the mountain villages of Peshkopia (Eastern Albania)," Journal of Mountain Science, vol. 11, no. 1, pp. 180-193, 2014.

[93] M. Shuaib, S. Ahmed, K. Ali et al., "Ethnobotanical and ecological assessment of plant resources at district dir, tehsil timergara, khyber pakhtunkhwa, Pakistan," Acta Ecologica Sinica, vol. 39, no. 1, pp. 109-115, 2019. 